IPPP-10-16

DCPT $-10-32$

arXiv:1003.4260

October 26, 2018

\title{
SUSY FLAVOR : a computational tool for FCNC and CP-violating processes in the MSSM
}

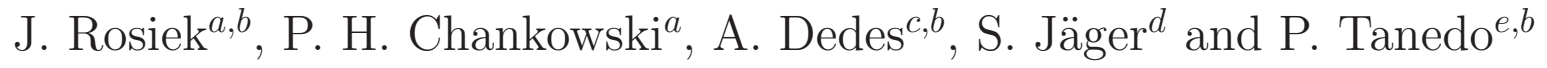 \\ ${ }^{a}$ Institute of Theoretical Physics, University of Warsaw, Hoża 69, 00-681 Warsaw, Poland \\ ${ }^{b}$ Institute for Particle Physics Phenomenology, University of Durham, DH1 3LE, UK \\ ${ }^{c}$ Division of Theoretical Physics, University of Ioannina, GR 45110, Greece \\ ${ }^{d}$ Department of Physics and Astronomy, University of Sussex, Brighton BN1 9QH, UK \\ ${ }^{e}$ Institute for High Energy Phenomenology, Newman Laboratory of Elementary Particle \\ Physics, Cornell University, Ithaca, NY 14853, USA
}

\begin{abstract}
We present SUSY_FLAVOR - a Fortran 77 program that calculates important leptonic and semi-leptonic low-energy observables in the general $R$-parity conserving MSSM. For a set of input MSSM parameters, the code gives predictions for the $\bar{K}^{0} K^{0}, \bar{D} D, \bar{B}_{d} B_{d}$ and $\bar{B}_{s} B_{s}$ mixing parameters; $B \rightarrow X_{s} \gamma, B_{s, d} \rightarrow l^{+} l^{-}, K_{L}^{0} \rightarrow \pi^{0} \bar{\nu} \nu$ and $K^{+} \rightarrow \pi^{+} \bar{\nu} \nu$ decay branching ratios; and the electric dipole moments of the leptons and the neutron. All these quantities are calculated at one-loop level (with some higher-order QCD corrections included) in the exact sfermion mass eigenbasis, without resorting to mass insertion approximations. The program can be obtained from http://www.fuw.edu.pl/susy_flavor.
\end{abstract}




\section{Introduction}

Flavor changing neutral currents (FCNCs) in the Minimal Supersymmetric Standard Model (MSSM) [1] originate from the fact that one cannot, in general, simultaneously diagonalize the mass matrices of fermions and their supersymmetric partners. The misalignment between these mass matrices leads to FCNCs at tree level. Moreover a large number of the MSSM parameters which can take complex values is a potential source of CP violation. Thus supersymmetric contributions to amplitudes of processes violating flavor and to quantities measuring CP violation, like Electric Dipole Moments (EDMs), can exceed by orders of magnitudes the ones of the SM particles. Such large effects are ruled out by experimental measurements which generally agree with the SM predictions and thus provide strong bounds on the amount of flavor and CP violation in the MSSM. For instance, measurements of the kaon system properties prohibit FCNC couplings between the first and second generation of down-type squarks larger than $10^{-3}$. These strong limits are often called the "SUSY flavor problem". However, there are also areas where current experiments still leave room for large SUSY contributions. For example, constraints from $B$-meson experiments allow an $\mathcal{O}(1)$ mixing between the second and third generation down-type squarks. Such a large mixing could produce FCNC effects that could be observed in the future at $B$-factories and/or hadron colliders, like the Tevatron and LHC [2].

Even assuming the so-called Minimal Flavor Violation (MFV) scenario in which all FCNC effects originate from the superpotential Yukawa couplings, the flavor conserving soft SUSY breaking parameters can still contain complex phases that cannot be absorbed by a redefinition of fields and can, for example, give large contributions to the electron and neutron EDMs.

As the accuracy of rare decay experiments improves, it is important to have a universal computational tool which would help compare new data with the predictions of the MSSM. Constructing such a tool is a non-trivial task because finding SUSY contribution to each rare decay requires tedious calculations, especially when one wishes to have fully general formulae that do not rely on the restrictive assumptions of the MFV scenario. Numerous analyses have been published in the literature, but because of the complexity of the problem, they mostly take into account one, or at most few, rare decays simultaneously. Furthermore, most analyses done for general flavor violation in the MSSM soft terms use the mass insertion approximation (MIA) (see e.g. [3]) which significantly simplifies calculations but does not produce correct results when flavor violation in the superpartner sector becomes strong.

In a series of papers since 1997 [3], many supersymmetric FCNC and CP-violating observables were analyzed with loop-level accuracy within the setup of the fully general $R$ parity conserving MSSM without resorting to any MIA-type expansions. A FORTRAN 77 computer programs based on the common set of Feynman rules of Ref. [14] were developed for each process. Because these programs use the same conventions, input parameters, and 


\begin{tabular}{|c|c|}
\hline Observable & Experiment \\
\hline \multicolumn{2}{|r|}{$\Delta F=0$} \\
\hline 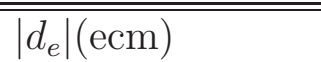 & $\overline{c<1.6 \times 10^{-27}[16]}$ \\
\hline$\left|d_{\mu}\right|(\mathrm{ecm})$ & $<2.8 \times 10^{-19}$ \\
\hline$\left|d_{\tau}\right|(\mathrm{ecm})$ & $<1.1 \times 10^{-17}[18$ \\
\hline$\left|d_{n}\right|(\mathrm{ecm})$ & $<2.9 \times 10^{-26}[19]$ \\
\hline \multicolumn{2}{|r|}{$\Delta F=1$} \\
\hline$\overline{\mathrm{Br}\left(K_{L} \rightarrow \pi^{0} \nu \nu\right)}$ & $\begin{array}{l}<6.7 \times 10^{-8}[20] \\
{[2]}\end{array}$ \\
\hline $\operatorname{Br}\left(K^{+} \rightarrow \pi^{+} \nu \nu\right)$ & $17.3_{-10.5}^{+11.5} \times 10^{-11}[21]$ \\
\hline $\operatorname{Br}\left(B_{d} \rightarrow e e\right)$ & $<1.13 \times 10^{-7} 22$ \\
\hline $\operatorname{Br}\left(B_{d} \rightarrow \mu \mu\right)$ & $<1.8 \times 10^{-8}[23]$ \\
\hline $\operatorname{Br}\left(B_{d} \rightarrow \tau \tau\right)$ & $<4.1 \times 10^{-3}[24]$ \\
\hline $\operatorname{Br}\left(B_{s} \rightarrow e e\right)$ & $<7.0 \times 10^{-5}[25]$ \\
\hline $\operatorname{Br}\left(B_{s} \rightarrow \mu \mu\right)$ & $<5.8 \times 10^{-8}[23]$ \\
\hline $\operatorname{Br}\left(B_{s} \rightarrow \tau \tau\right)$ & -- \\
\hline $\operatorname{Br}\left(B \rightarrow X_{s} \gamma\right)$ & $(3.52 \pm 0.25) \times 10^{-4}[26$ \\
\hline \multicolumn{2}{|r|}{$\Delta F=2$} \\
\hline$\overline{\mid \overline{\left|\epsilon_{K}\right|}}$ & $(2.229 \pm 0.010) \times 10^{-3}[18$ \\
\hline$\Delta M_{K}$ & $(5.292 \pm 0.009) \times 10^{-3} \mathrm{ps}^{-1}[18$ \\
\hline$\Delta M_{D}$ & $\left(2.37_{-0.71}^{+0.66}\right) \times 10^{-2} \mathrm{ps}^{-1}[18$ \\
\hline$\Delta M_{B_{d}}$ & $(0.507 \pm 0.005) \mathrm{ps}^{-1}[26]$ \\
\hline$\Delta M_{B_{s}}$ & $(17.77 \pm 0.12) \mathrm{ps}^{-1}[27]$ \\
\hline
\end{tabular}

Table 1: List of observables calculated by SUSY_FLAVOR and their currently measured values or $95 \%$ C.L bounds (except for $\operatorname{Br}\left(B_{d} \rightarrow e e\right)$ and $\operatorname{Br}\left(B_{d} \rightarrow \tau \tau\right)$ for which the $90 \%$ C.L bounds are given.).

internal data structures, they can naturally interface with one another. Combining these works, we present in this article SUSY_FLAVOR - a publicly available computer code that simultaneously calculates the set of important $\Delta F=0,1,2 \mathrm{FCNC}$ and CPV observables in the framework of the general MSSM. The current version (1.0) of the program takes a set of MSSM parameters and calculates the processe 1 listed in Table 1 .

Several programs allowing to analyze various aspects of the MSSM flavor phenomenology have been published. The most relevant to SUSY_FLAVOR are: CPsuperH [28], SusyBSG [29] and SuperIso [30]. SusyBSG is dedicated to high-precision predictions for $B \rightarrow s \gamma$ while CPsuperH and SuperIso calculate processes similar to the ones computed by SUSY_FLAVOR.

\footnotetext{
${ }^{1}$ In the current version SUSY_FLAVOR calculates also the full one-loop corrections to lepton flavor violating $B$-meson decays such as $B \rightarrow \mu \tau$. However, it is known that contributions to the amplitudes of these processes are greatly enhanced at large $\tan \beta$ by formally two loop double penguin diagrams [15] which currently are not included in the code. Thus, SUSY_FLAVOR can be used to estimate such decay rates only at low $\tan \beta \lesssim 10$.
} 
However, these existing codes are restricted to the Minimal Flavor Violation scenario. Thus, to the best of the authors' knowledge, SUSY_FLAVOR is the first program which can simultaneously calculate the set of rare decays listed in Table 1 without any (apart from the $R$-parity conservation) restrictions on the choice of MSSM parameters. Other publicly available codes that are relevant to SUSY_FLAVOR (which can e.g. calculate the MSSM soft parameters used as input to SUSY_FLAVOR, or for the same set of input parameters calculate non-FCNC related observables) are FeynHiggs [31], SoftSUSY [32], SuSpect [33], SPheno [34], MicrOMEGAs [35], DarkSUSY [36] and NMHDECAY [37].

In summary, the basic features of SUSY_FLAVOR are:

- The program utilizes the most general $R$-parity conserving Lagrangian for the MSSM. In addition to standard soft breaking terms, it can even accommodate additional nonholomorphic terms, such as

$$
A_{d}^{\prime I J} H_{i}^{2 \star} Q_{i}^{I} D^{J}+A_{u}^{\prime I J} H_{i}^{1 \star} Q_{i}^{I} U^{J}+\text { H.c. , }
$$

that, for example, do not appear in the minimal supergravity scenario but are present in the most general softly broken supersymmetric effective Lagrangian [38].

- There is no limit on the size of flavor violating parameters because the calculation does not rely on the MIA expansion. Complex "mass insertions" of the form

$$
\delta_{Q X Y}^{I J}=\frac{\left(M_{Q}^{2}\right)_{X Y}^{I J}}{\sqrt{\left(M_{Q}^{2}\right)_{X X}^{I I}\left(M_{Q}^{2}\right)_{Y Y}^{J J}}},
$$

$(I, J$ denote quark flavors, $X, Y$ denote superfield chirality, and $Q$ indicates either the up or down quark superfield sector, similarly for slepton superfields) are taken as inputs, but they only serve to conveniently parametrize the sfermion mass matrices. SUSY_FLAVOR numerically calculates the exact tree-level spectrum and mixing matrices, which are later used in loop calculations.

- As an intermediate step, parton-level form factors for quark and lepton 2-, 3- and 4-point Green functions are calculated. They are later dressed in hadronic matrix elements (see Table 3 in Section 3) to obtain predictions for the physical quantities listed in Table 1. The set of Green's functions computed by SUSY_FLAVOR as intermediate "building blocks" is quite universal and can be used by other authors to calculate other processes.

- The program runs fairly quickly. On a Mac PowerBook G4 with GNU FORTRAN g77 it returns the output for a single parameter set run within a second.

We note that the current SUSY_FLAVOR version does not resum higher-order corrections in the limit of large $\tan \beta$. Such corrections in the MFV scenario can easily dominate the SM result for $\tan \beta \gtrsim 30-40$. However, even in that case new sources of flavor violation often give comparable or more significant contributions than the MFV-type tan $\beta$-enhanced 
corrections. Thus, for large $\tan \beta$ one should perform the resummation of leading higherorder terms in the presence of the non-vanishing flavor violation in the sfermion mass matrices. Unfortunately, such resummation is not yet fully understood, i.e., although there are theoretical ideas [10, 39 41] on how to resum these contributions beyond MFV, it is quite difficult to implement them into SUSY_FLAVOR without losing numerical stability for large mass insertions. Thus, even though some parts of SUSY_FLAVOR (e.g. $\bar{B} B$ mixing and the $B \rightarrow l^{+} l^{-}$decays) were originally devised to perform a large $\tan \beta$ resummation for the MSSM parameter choice restricted to the MFV case, in the present version this option has been deactivated for consistency. We hope to improve this in future versions of the code. The current version of SUSY_FLAVOR should be used for low to moderate values of $\tan \beta(\tan \beta \lesssim 30)$, however even in the case of significant supersymmetric flavor violation it should still produce reasonably accurate results even for $\tan \beta$ beyond this range.

The rest of the paper is organized as follows. In Section 2 we define the general structure of the MSSM Lagrangian following Ref. [14] to facilitate comparison of the conventions used in SUSY_FLAVOR with others used in the literature and to connect the variables used in the code with physical quantities. Section 3 describes the internal structure of SUSY_FLAVOR, the most important steps of calculations, and the file structure of the library. In Section 4 we carefully present the initialization sequence for SUSY_FLAVOR, defining input parameters and how they are used. Routines for calculating the FCNC and CPV observables collected in Table 1 are described in Section 5. We conclude with a summary of the presentation. Appendix A contains brief instructions on how to install and run the SUSY_FLAVOR package. In Appendices $\mathrm{B}$ and $\mathrm{C}$ we provide templates for initializing SUSY_FLAVOR from within the program and using an external file in the SLHA2 format [42], respectively. Both of these templates produce the set of test results listed in Appendix D.

SUSY_FLAVOR can be downloaded from the following address:

http://www.fuw.edu.pl/susy_flavor

\section{Lagrangian, conventions and the tree level masses}

\subsection{Lagrangian parameters}

SUSY_FLAVOR follows the conventions for the MSSM Lagrangian and Feynman rules for the most general $R$-parity conserving version of the MSSM Lagrangian given in [14]. Over 100 Lagrangian parameters are taken as input to SUSY_FLAVOR and can be initialized independently.

For completeness and for easier comparison with conventions used in other sources, we present here the full list of the general MSSM couplings. They can be classified by sectors of the theory: 
1. Gauge sector. $g_{1}, g_{2}, g_{3}$ denote the coupling constants of gauge groups $U(1)_{Y}, S U(2)_{L}$, $S U(3)_{c}$, respectively.

2. Superpotential and Yukawa couplings. The superpotential and the soft breaking sfermion couplings are written after the rotations of superfields to the super-KM basis in which the Yukawa couplings are diagonal and the soft parameters are redefined accordingly to account for accommodate these flavor rotations (see e.g. [3]).

We do not assume the existence of the heavy right-handed neutrino/sneutrino supermultiplet and neglect related terms in the Lagrangian? 2 Then the most general form of the $R$-parity conserving MSSM superpotential takes the form:

$$
W=\mu \epsilon_{i j} H_{i}^{1} H_{j}^{2}+\epsilon_{i j} Y_{l}^{I} H_{i}^{1} L_{j}^{I} R^{I}+\epsilon_{i j} Y_{d}^{I} H_{i}^{1} Q_{j}^{I} D^{I}+\epsilon_{i j} Y_{u}^{I} H_{i}^{2} Q_{j}^{I} U^{I} .
$$

Capital indices $(I, J, K \ldots)$ label matter field generations and run from 1 to 3 . Lower-case indices $(i, j, \ldots)$ are $S U(2)_{L}$ indices (we use $\epsilon_{12}=-1$ ). $S U(3)_{c}$ indices are not written explicitly; we assume that the $Q$ supermultiplets are QCD triplets and the $D$ and $U$ supermultiplets are anti-triplets. At tree level, quark and lepton masses are related to Yukawa couplings by (note that $Y_{l}^{I}, Y_{d}^{I}$ are negative in our convention):

$$
m_{e}^{I}=-\frac{v_{1} Y_{l}^{I}}{\sqrt{2}}, \quad m_{d}^{I}=-\frac{v_{1} Y_{d}^{I}}{\sqrt{2}}, \quad m_{u}^{I}=\frac{v_{2} Y_{u}^{I}}{\sqrt{2}} .
$$

It follows that in SUSY_FLAVOR the fermion masses and the elements of the CabibboKobayashi-Maskawa (CKM) matrix, rather than the Yukawa couplings, are used as input parameters.

3. Soft gaugino mass terms for the $U(1)_{Y}, S U(2)_{L}$ and $S U(3)_{c}$ gauge groups

$$
\frac{1}{2} M_{1} \lambda_{B} \lambda_{B}+\frac{1}{2} M_{2} \lambda_{A}^{i} \lambda_{A}^{i}+\frac{1}{2} M_{3} \lambda_{G}^{a} \lambda_{G}^{a}+\text { h.c. }
$$

\section{Soft-breaking mass terms for the scalar fields.}

$$
\begin{gathered}
-m_{H_{1}}^{2} H_{i}^{1 \star} H_{i}^{1}-m_{H_{2}}^{2} H_{i}^{2 \star} H_{i}^{2}-\left(m_{L}^{2}\right)^{I J} L_{i}^{I \star} L_{i}^{J}-\left(m_{E}^{2}\right)^{I J} E^{I \star} E^{J} \\
-\left(K m_{Q}^{2} K^{\dagger}\right)^{I J} Q_{1}^{I \star} Q_{1}^{J}-\left(m_{Q}^{2}\right)^{I J} Q_{2}^{I \star} Q_{2}^{J}-\left(m_{D}^{2}\right)^{I J} D^{I \star} D^{J}-\left(m_{U}^{2}\right)^{I J} U^{I \star} U^{J}
\end{gathered}
$$

5. Trilinear scalar couplings corresponding to superpotential Yukawa terms.

$$
m_{12}^{2} \epsilon_{i j} H_{i}^{1} H_{j}^{2}+\epsilon_{i j} A_{l}^{I J} H_{i}^{1} L_{j}^{I} E^{J}+\epsilon_{i j} A_{d}^{I J} H_{i}^{1} Q_{j}^{I} D^{J}+\epsilon_{i j} A_{u}^{I J} H_{i}^{2} Q_{j}^{I} U^{J}+\text { h.c. }
$$

6. Non-standard trilinear scalar couplings involving complex conjugated Higgs fields (sometimes called "non-analytic terms").

$$
A_{l}^{\prime I J} H_{i}^{2 \star} L_{i}^{I} E^{J}+A_{d}^{\prime I J} H_{i}^{2 \star} Q_{i}^{I} D^{J}+A_{u}^{\prime I J} H_{i}^{1 \star} Q_{i}^{I} U^{J}+\text { h.c. }
$$

\footnotetext{
${ }^{2}$ The modifications to the phenomenology of the MSSM from the presence of a heavy right neutrino supermultiplet are discussed in [43. Some numerical codes concerning the problem can be obtained from its authors.
} 
Usually these couplings are not considered as they are not generated in standard SUSY breaking models. However, for completeness they are included in SUSY_FLAVOR and by default initialized to zero. Users of SUSY_FLAVOR may decide to set them to some nonvanishing values in order to check their impact on rare decay phenomenology.

In general, the mass parameters $\mu, m_{12}^{2}, m_{Q}^{2}, M_{1,2,3}$, and the trilinear soft couplings may be complex. Global rephasing of all fermion fields of the theory and of one of the Higgs multiplets can render two of these parameters real [4]. We choose them to be the gluino mass $M_{3}$ and the soft Higgs mixing term $m_{12}^{2}$. The latter choice keeps the Higgs vacuum expectation values (VEV) and, therefore, the parameter $\tan \beta$ real at tree level.

\subsection{Physical tree level masses and mixing angles}

Mass matrices of the MSSM particles can be written in terms of the parameters of Section 2.1. In SUSY_FLAVOR, following [14], we consistently use matrix notation for all fields, including neutral and charged Higgs bosons. Such a notation simplifies the expressions for loop calculations. In this Section we explicitly write down all mass matrices to fix our sign conventions relative to other choices in the literature.

1. Higgs sector. We denote the CP-even and CP-odd neutral scalars as $H_{i}^{0}$ and $A_{i}^{0}$, respectively, with $i=1,2$. In terms of more common notation, $\left(H_{1}^{0}, H_{2}^{0}\right) \equiv\left(H^{0}, h^{0}\right)$ and $\left(A_{1}^{0}, A_{2}^{0}\right) \equiv\left(A^{0}, G^{0}\right)$. These are related to the initial Higgs doublets by (no sum over $i$ ):

$$
\begin{aligned}
\Re \mathrm{e} H_{i}^{i} & =\frac{1}{\sqrt{2}}\left(Z_{R}^{i j} H_{j}^{0}+v_{i}\right), \\
\Im \mathrm{m} H_{i}^{i} & =\frac{1}{\sqrt{2}} Z_{H}^{i j} A_{j}^{0} .
\end{aligned}
$$

In these formulae $v_{1}, v_{2}$ are the VEVs of the two neutral components of the Higgs doublets and $Z_{R}, Z_{H}$ are the mixing matrices in the CP-even and CP-odd Higgs sectors, respectively.

The mixing matrix $Z_{R}$ and the masses of $H_{i}^{0}$ can be obtained by diagonalizing the CP-even Higgs mass $^{2}$ matrix:

$$
Z_{R}^{T}\left(\begin{array}{cc}
-m_{12}^{2} \frac{v_{2}}{v_{1}}+\frac{e^{2} v_{1}^{2}}{4 s_{W}^{2} c_{W}^{2}} & m_{12}^{2}-\frac{e^{2} v_{1} v_{2}}{4 s_{W}^{2} c_{W}^{2}} \\
m_{12}^{2}-\frac{e^{2} v_{1} v_{2}}{4 s_{W}^{2} c_{W}^{2}} & -m_{12}^{2} \frac{v_{1}}{v_{2}}+\frac{e^{2} v_{2}^{2}}{4 s_{W}^{2} c_{W}^{2}}
\end{array}\right) Z_{R}=\left(\begin{array}{cc}
M_{H_{1}^{0}}^{2} & 0 \\
0 & M_{H_{2}^{0}}^{2}
\end{array}\right) \equiv\left(\begin{array}{cc}
M_{H}^{2} & 0 \\
0 & M_{h}^{2}
\end{array}\right) .
$$

$A_{1}^{0}\left(\equiv A^{0}\right)$ has mass $M_{A}^{2}=m_{H_{1}}^{2}+m_{H_{2}}^{2}+2|\mu|^{2}$. SUSY_FLAVOR assumes $R_{\xi}$ gauge with $\xi=1$, so the neutral Goldstone boson $A_{2}^{0}\left(\equiv G^{0}\right)$ has the mass $M_{G^{0}}=M_{Z}$.

The mixing matrices $Z_{H}, Z_{R}$ are parametrized as follows:

$$
Z_{H}=\left(\begin{array}{cc}
\sin \beta & -\cos \beta \\
\cos \beta & \sin \beta
\end{array}\right), \quad Z_{R}=\left(\begin{array}{cc}
\cos \alpha & -\sin \alpha \\
\sin \alpha & \cos \alpha
\end{array}\right)
$$


with the angles $\alpha$ and $\beta$ determined by

$$
\begin{aligned}
\tan \beta=\frac{v_{2}}{v_{1}}, & 0 \leq \beta \leq \frac{\pi}{2}, \\
\tan 2 \alpha=\tan 2 \beta \frac{M_{A}^{2}+M_{Z}^{2}}{M_{A}^{2}-M_{Z}^{2}}, & -\frac{\pi}{2} \leq \alpha \leq 0 .
\end{aligned}
$$

Charged Higgs scalars are denoted by $H_{i}^{ \pm} \equiv H^{ \pm}, G^{ \pm}$and are related to the initial Higgs doublet again by the matrix $Z_{H}$ :

$$
\left(\begin{array}{c}
H_{2}^{1 \star} \\
H_{1}^{2}
\end{array}\right)=Z_{H}\left(\begin{array}{c}
H_{1}^{+} \\
H_{2}^{+}
\end{array}\right) .
$$

The physical charged Higgs boson has mass

$$
M_{H_{1}^{ \pm}}^{2}=M_{W}^{2}+m_{H_{1}}^{2}+m_{H_{2}}^{2}+2|\mu|^{2},
$$

while the charged Goldstone bosons $G^{ \pm}$have masses $M_{G^{ \pm}}=M_{W}$.

2. Gaugino sector. The chargino masses and mixing matrices $Z_{+}$and $Z_{-}$are defined by the relation

$$
\left(Z_{-}\right)^{T}\left(\begin{array}{cc}
M_{2} & \frac{e v_{2}}{\sqrt{2} s_{W}} \\
\frac{e v_{1}}{\sqrt{2} s_{W}} & \mu
\end{array}\right) Z_{+}=\left(\begin{array}{cc}
M_{\chi_{1}} & 0 \\
0 & M_{\chi_{2}}
\end{array}\right) .
$$

In SUSY_FLAVOR we choose $Z_{-}, Z_{+}$such that both masses $M_{\chi_{i}}$ are real positive and $M_{\chi_{2}}>$ $M_{\chi_{1}}$.

The neutralino tree level masses are given by

$$
Z_{N}^{T}\left(\begin{array}{cccc}
M_{1} & 0 & \frac{e v_{1}}{2 c_{W}} & \frac{e v_{2}}{2 C_{W}} \\
0 & M_{2} & \frac{e v_{1}}{2 s_{W}} & \frac{e v_{2}}{2 s_{W}} \\
\frac{-e v_{1}}{2 c_{W}} & \frac{e v_{1}}{2 s_{W}} & 0 & -\mu \\
\frac{e v_{2}}{2 c_{W}} & \frac{-e v_{2}}{2 s_{W}} & -\mu & 0
\end{array}\right) Z_{N}=\left(\begin{array}{ccc}
M_{\chi_{1}^{0}} & & 0 \\
& \ddots & \\
0 & & M_{\chi_{4}^{0}}
\end{array}\right),
$$

where again we use the ambiguity in the definition of the $Z_{N}$ matrix to choose to make all $M_{\chi_{i}^{0}}$ real positive and increasingly ordered.

3. Slepton sector. The three complex sneutrino fields have tree level masses and the mixing matrix $Z_{\tilde{\nu}}$ defined by:

$$
Z_{\tilde{\nu}}^{\dagger}\left(\frac{e^{2}\left(v_{1}^{2}-v_{2}^{2}\right)}{8 s_{W}^{2} c_{W}^{2}} \hat{1}+m_{L}^{2}\right) Z_{\tilde{\nu}}=\left(\begin{array}{ccc}
M_{\tilde{\nu}_{1}}^{2} & & 0 \\
& \ddots & \\
0 & & M_{\tilde{\nu}_{3}}^{2}
\end{array}\right) .
$$

The mass matrix for the six charged sleptons

$$
\mathcal{M}_{L}^{2}=\left(\begin{array}{cc}
\frac{e^{2}\left(v_{1}^{2}-v_{2}^{2}\right)\left(1-2 c_{W}^{2}\right)}{8 s_{W}^{2} c_{W}^{2}}+\frac{v_{1}^{2} Y_{l}^{2}}{2}+\left(m_{L}^{2}\right)^{T} & \frac{v_{2}}{\sqrt{2}}\left(Y_{l} \mu^{\star}-A_{l}^{\prime}\right)+\frac{v_{1}}{\sqrt{2}} A_{l} \\
\frac{v_{2}}{\sqrt{2}}\left(Y_{l} \mu-A_{l}^{\dagger}\right)+\frac{v_{1}}{\sqrt{2}} A_{l}^{\dagger} & -\frac{e^{2}\left(v_{1}^{2}-v_{2}^{2}\right)}{4 c_{W}^{2}}+\frac{v_{1}^{2} Y_{l}^{2}}{2}+m_{E}^{2}
\end{array}\right)
$$


is diagonalized by the unitary matrix $Z_{L}$,

$$
Z_{L}^{\dagger} \mathcal{M}_{L}^{2} Z_{L}=\left(\begin{array}{ccc}
M_{L_{1}}^{2} & & 0 \\
& \ddots & \\
0 & & M_{L_{6}}^{2}
\end{array}\right)
$$

4. Squark sector. Analogously, for the up and down squarks one has:

$$
\begin{aligned}
& \mathcal{M}_{U}^{2}=\left(\begin{array}{cc}
\frac{e^{2}\left(v_{1}^{2}-v_{2}^{2}\right)\left(4 c_{W}^{2}-1\right)}{24 s_{W}^{2} c_{W}^{2}}+\frac{v_{2}^{2} Y_{u}^{2}}{2}+\left(K m_{Q}^{2} K^{\dagger}\right)^{T} & -\frac{v_{1}}{\sqrt{2}}\left(Y_{u} \mu^{\star}+A_{u}^{\prime}\right)-\frac{v_{2}}{\sqrt{2}} A_{u} \\
-\frac{v_{1}}{\sqrt{2}}\left(Y_{u} \mu+A_{u}^{\dagger}\right)-\frac{v_{2}}{\sqrt{2}} A_{u}^{\dagger} & \frac{e^{2}\left(v_{1}^{2}-v_{2}^{2}\right)}{6 c_{W}^{2}}+\frac{v_{2}^{2} Y_{u}^{2}}{2}+m_{U}^{2}
\end{array}\right) \\
& Z_{U}^{T} \mathcal{M}_{U}^{2} Z_{U}^{\star}=\left(\begin{array}{ccc}
M_{U_{1}}^{2} & & 0 \\
& \ddots & \\
0 & & M_{U_{6}}^{2}
\end{array}\right) \\
& \mathcal{M}_{D}^{2}=\left(\begin{array}{cc}
-\frac{e^{2}\left(v_{1}^{2}-v_{2}^{2}\right)\left(1+2 c_{W}^{2}\right)}{24 s_{W}^{2} c_{W}^{2}}+\frac{v_{1}^{2} Y_{d}^{2}}{2}+\left(m_{Q}^{2}\right)^{T} & \frac{v_{2}}{\sqrt{2}}\left(Y_{d} \mu^{\star}-A_{d}^{\prime}\right)+\frac{v_{1}}{\sqrt{2}} A_{d} \\
\frac{v_{2}}{\sqrt{2}}\left(Y_{d} \mu-A_{d}^{\dagger}\right)+\frac{v_{1}}{\sqrt{2}} A_{d}^{\dagger} & -\frac{e^{2}\left(v_{1}^{2}-v_{2}^{2}\right)}{12 c_{W}^{2}}+\frac{v_{1}^{2} Y_{d}^{2}}{2}+m_{D}^{2}
\end{array}\right), \\
& Z_{D}^{\dagger} \mathcal{M}_{D}^{2} Z_{D}=\left(\begin{array}{ccc}
M_{D_{1}}^{2} & & 0 \\
& \ddots & \\
0 & & M_{D_{6}}^{2}
\end{array}\right)
\end{aligned}
$$

Note that $Z_{U}$ is defined with a complex conjugate compared to the definitions of $Z_{L}$ and $Z_{D}$. Thus all positively charged sfermion mass eigenstates are multiplied by $Z_{X}^{i j}$, while negatively charged eigenstates are multiplied by $Z_{X}^{i j \star}$.

\subsection{Interfacing with the Les Houches Accord}

SUSY_FLAVOR has been in development since 1996, long before the Les Houches Accord [44] (SLHA) for common MSSM Lagrangian conventions was agreed. Because of that, it was not feasible to change the internal SUSY_FLAVOR structure as it would require careful checking and rewriting of thousands of lines of a complicated code. Therefore we have decided to keep the conventions of [14] for the internal calculations in SUSY_FLAVOR. In Table 2 we summarize the differences of our conventions and those of the latest extended SLHA 2 [42]. These differences are quite minor and translation can be done by changing few signs and/or transposing matrices in the soft SUSY breaking sector. Thus, for the input parameters of SUSY_FLAVOR we leave the choice of convention as a user-defined option. 


\begin{tabular}{|c|c|}
\hline SLHA 2 [42] & Ref. [14] \\
\hline$\hat{T}_{U}, \hat{T}_{D}, \hat{T}_{E}$ & $-A_{u}^{T},+A_{d}^{T},+A_{l}^{T}$ \\
$\hat{m}_{\tilde{Q}}^{2}, \hat{m}_{\tilde{L}}^{2}$ & $m_{Q}^{2}, m_{L}^{2}$ \\
$\hat{m}_{\tilde{u}}^{2}, \hat{m}_{\tilde{d}}^{2}, \hat{m}_{\tilde{I}}^{2}$ & $\left(m_{U}^{2}\right)^{T},\left(m_{D}^{2}\right)^{T},\left(m_{E}^{2}\right)^{T}$ \\
$\mathcal{M}_{\tilde{u}}^{2}, \mathcal{M}_{\tilde{d}}^{2}$ & $\left(\mathcal{M}_{U}^{2}\right)^{T},\left(\mathcal{M}_{D}^{2}\right)^{T}$ \\
\hline
\end{tabular}

Table 2: Comparison of SLHA [42] and Ref. [14] conventions.

Currently SUSY_FLAVOR does not use the super-PMNS basis for the lepton and slepton sector; only the charged lepton Yukawa matrix (and not the neutrino mass matrix) is diagonalized. The super-PMNS basis can become helpful once new experiments are able to identify the flavor of the neutrinos produced in rare decays, but at present this is not experimentally feasible.

\section{Structure of the code}

Calculations in SUSY_FLAVOR take the following steps:

1. Parameter initialization. This is the most important step for SUSY_FLAVOR users and is described in detail Section 4. Users can adjust the basic Standard Model parameters according to latest experimental data and initialize all (or the chosen subset of) supersymmetric soft masses and couplings and Higgs sector parameters listed in Section 2.1.

2. Calculation of the physical masses and the mixing angles. After setting the input parameters, SUSY_FLAVOR calculates the eigenvalues of the mass matrices of all MSSM particles and their mixing matrices at the tree level. Diagonalization is done numerically without any approximations.

3. Calculation of Wilson coefficients at the SUSY scale. Physical tree-level masses and mixing matrices are used to evaluate exact one-loop Wilson coefficients of the effective operators required for a given process. Again, the formulae used in the code are exact, i.e. do not rely on any approximations, such as the MIA expansion. In the current version, SUSY_FLAVOR calculates Wilson coefficients generated by the diagrams listed in Table 3, All Wilson coefficients are calculated at the high energy scale, assumed to be the average mass of SUSY particles contributing to a given process or the top quark scale.

It is important to stress that SUSY_FLAVOR accepts fermion generation indices and Higgs boson indices as input parameters. Thus in Table $3 d$ and $u, l$ and $\nu$ denote quarks or leptons of any generation and, similarly, $H_{i}^{0}$ and $A_{i}^{0}$ denote any type of the neutral Higgs bosons. Hence, the actual number of amplitudes which can be calculated using combinations of these form factors is much larger than needed for the rare decay rates currently implemented fully in SUSY_FLAVOR. We plan to add new processes in future releases of our library. 


\begin{tabular}{|c|c|c|}
\hline Box & Penguin & Self energy \\
\hline \hline$d d d d$ & $Z \bar{d} d, \gamma \bar{d} d, g \bar{d} d$ & $d$-quark \\
uиuu & $H_{i}^{0} \bar{d} d, A_{i}^{0} \bar{d} d$ & $u$-quark \\
$d d l l$ & $H_{i}^{0} \bar{u} u, A_{i}^{0} \bar{u} u$ & \\
$d d \nu \nu$ & & \\
\hline
\end{tabular}

Table 3: One loop parton level diagrams implemented in SUSY_FLAVOR.

4. Strong corrections. In its final step SUSY_FLAVOR performs (when necessary) the QCD evolution of Wilson coefficients from the high energy (SUSY or top quark mass) scale to the low energy scale appropriate for a given rare decay, calculates the relevant hadronic matrix elements, and outputs predictions for physical quantities. The formulae for QCD and hadronic corrections are primarily based on calculations performed in the SM and supplemented, when necessary, with contributions from non-standard operators which usually are neglected in the SM, because they are suppressed by powers of the light quark Yukawa couplings. This part of SUSY_FLAVOR is based on analyses published by other authors, whereas points 1-3 are implemented using our own calculations. The accuracy of strong corrections differ from process to process, from negligible or small (leptonic EDM, "gold-plated" decay modes $K \rightarrow \pi \bar{\nu} \nu$ [45]) to order of magnitude uncertainties (unknown long distance contributions to $\Delta m_{K}$ or $\Delta m_{D}$ ). Even in the case of large QCD uncertainties, the result of the calculation performed by SUSY_FLAVOR can be of some use. Flavor violation in the sfermion sector can lead to huge modifications of many observables, sometimes by several orders of magnitude, so that comparison with experimental data can help to constrain the soft flavor-violating terms even if strong corrections are not very well known.

Below we list the files included in the SUSY_FLAVOR library with a brief description of their content and purpose.

eisch1.f: auxiliary numerical routine - hermitian matrix diagonalization

vegas.f: auxiliary numerical routine - Vegas Monte Carlo integration

rombint.f: auxiliary numerical routine - Romberg numerical integration

sflav_io.f: input routine for reading of the SLHA2 format; test output routines

b_fun.f: general 2-point loop functions

db_fun.f: derivatives of general 2-point loop functions

c_fun.f: general 3-point loop functions

cd_fun.f : 3-, 4- and some 5-point loop functions at vanishing external momenta

vh_def.f: definitions of Higgs boson tree-level vertices

vg_def.f: definitions of gauge boson tree-level vertices 
$v f \_d e f . f$ definitions of fermion tree-level vertices

mh_init.f : initialization of MSSM parameters

mh_diag.f : diagonalization of tree level mass matrices; outputs physical masses and mixing angles qcd_fun.f: auxiliary QCD calculations - running $\alpha_{s}$, running quark masses etc.

d_self0.f : $d$-quark self-energy

u_self0.f: $u$-quark self-energy

sff_fun0.f: form factors of the general scalar-fermion-fermion 1-loop triangle diagram

sdd_vert0.f : CP-even Higgs-d quark-d quark 1-loop triangle diagram

pdd_vert0.f : CP-odd Higgs-d quark- $d$ quark 1-loop triangle diagram

suu_vert0.f: CP-even Higgs- $u$ quark- $u$ quark 1-loop triangle diagram

puu_vert0.f: CP-odd Higgs-u quark-u quark 1-loop triangle diagram

zdd_vert0.f : $Z$ boson- $d$ quark- $d$ quark 1-loop triangle diagram

ddg_fun.f : form factors for the general gauge boson-fermion-fermion 1-loop triangle diagram

dd_gluon.f : $d$ quark- $d$ quark-gluon 1-loop triangle diagram

dd_gamma.f : $d$ quark- $d$ quark-photon 1-loop triangle diagram

bsg_nl.f: formulae for $\operatorname{Br}\left(B \rightarrow X_{s} \gamma\right)$, including QCD corrections

dd_ll.f : $d$ quark- $d$ quark-lepton-lepton 1-loop box diagram

dd_vv.f : $d$ quark-d quark-neutrino-neutrino 1-loop box diagram

phen_2q.f : formulae for $\operatorname{Br}\left(K_{L}^{0} \rightarrow \pi^{0} \bar{\nu} \nu\right), \operatorname{Br}\left(K^{+} \rightarrow \pi^{+} \bar{\nu} \nu\right)$ and $\operatorname{Br}\left(B_{s(d)} \rightarrow l^{+} l^{-}\right)$including QCD corrections and hadronic matrix elements

dd_mix.f : 4-d quark 1-loop box diagram

uu_mix.f : 4-u quark 1-loop box diagram

phen_4q.f: formulae for the meson mixing observables: $\Delta m_{K}, \epsilon_{K}, \Delta m_{D}, \Delta m_{B_{d(s)}}$ including QCD corrections and hadronic matrix elements

edm_l.f: lepton electric dipole moment

cdm_d.f : $d$-quark chromoelectric dipole moment

cdm_u.f : $u$-quark chromoelectric dipole moment

cdm_g.f: gluon chromoelectric dipole moment

edm_d.f : $d$-quark electric dipole moment 
edm_u.f : $u$-quark electric dipole moment

edm_n.f : $u$-quark electric dipole moment

All the 2-, 3- and 4-point Green functions are calculated for vanishing external momenta. As mentioned before, by " $u$ quark" and " $d$ quark" we mean all generations of quarks.

In addition to the files listed above, the library contains the master driver file susy_flavor.f which illustrates the proper initialization sequence for SUSY_FLAVOR parameters and produces a set of test results for the implemented observables.

\section{Parameter initialization in SUSY_FLAVOR}

We now list the input parameters used by SUSY_FLAVOR. These are not always directly the MSSM Lagrangian parameters given in Section 2.1 - for example, instead of using the $\mu$ parameter and the soft Higgs masses $m_{H_{i}}^{2}, m_{12}^{2}$, it is customary to use $\tan \beta$ and the CPodd Higgs mass $M_{A}$ to parametrize MSSM Higgs sector. In its first step, SUSY_FLAVOR restores the Lagrangian parameters of Section 2.1 for the given set of more human-friendly input parameters. Then, the remaining routines use the "raw" Lagrangian parameters - if necessary they can also be directly modified by (experienced!) users.

In the rest of this section we describe step-by-step the basic initialization routines used by SUSY_FLAVOR, their arguments and, when necessary, the FORTRAN common blocks storing the most important data (other common blocks serve for the internal purposes and usually do not need to be accessed by users).

By default, SUSY_FLAVOR uses the following implicit type declaration in all routines:

implicit double precision ( $a-h, o-z)$

so that all variables in SUSY_FLAVOR with the names starting from a to $\mathrm{h}$ and from o to $\mathrm{z}$ are automatically defined as double precision and those with names starting from $\mathrm{i}$ to $\mathrm{n}$ are of integer type. In what follows we explicitly indicate variables that do not obey this rule. Such variables are always listed in explicit type statements inside the procedures. Complex parameters mentioned in this article are declared in SUSY_FLAVOR as double complex type. Mass parameters are always given in $\mathrm{GeV}$.

SUSY_FLAVOR provides two ways of initializing input parameters. As the first option, they can be read from the file susy_flavor. in. The structure of this file follows the SLHA2 convention [42], with some extensions which we describe in Section 4.1. Initializing parameters in the input file is simple, it is done by a call to single subroutine sflav_input and does not require detailed knowledge of the program internal structure. This option is particularly convenient for testing a single parameter set but can be cumbersome for scans over the MSSM parameter space. Therefore, as a second option, SUSY_FLAVOR also provides a set of routines designed to initialize parameters defined in the program, which can easily be used 
to prepare programs that scan over large parameter sets. As described in Section 4.2, these routines require more care in use, as they should be initialized in the proper order, i.e. the gauge sector first, then the fermion sector, Higgs sector, and SUSY sectors at the end (the initialization sequences for the gaugino, slepton and squark sectors are independent).

An example of a full initialization sequence for SUSY_FLAVOR, illustrating both options mentioned above, is presented Appendix B. The sample input file susy_flavor.in is given in Appendix C. Test output generated for parameters used in Appendices B and C is enclosed in Appendix D.

\subsection{Parameter initialization from the input file}

Input parameters for SUSY_FLAVOR can be set by editing appropriate entries of the file susy_flavor.in and subsequently calling the subroutine sflav_input, which reads the input file, stores the the MSSM Lagrangian parameters in FORTRAN common blocks and calculates tree-level physical masses and mixing matrices. After calling sflav_input, all physical observable described in Section 5 can be calculated.

The input file susy_flavor. in is written in the SLHA2 format, with some extensions which we list below (for an example of a complete input file see Appendix C).

1. We define a non-standard Block SOFTINP. Currently it contains two control variables, iconv and input_type. These serve to choose input conventions in the sfermion sector (in other sectors SLHA2 and Ref. [14] agree).

Variable value Sfermion sector parametrization

$i \operatorname{conv}=1 \quad$ MSSM parameters defined in SLHA 2 conventions.

iconv $=2 \quad$ MSSM parameters defined in conventions of Ref. [14].

input_type $=1 \quad$ sfermion diagonal trilinear mixing terms given as dimensionless parameters; all off-diagonal soft terms are given as dimensionless mass insertions - see comments below on the data blocks defining the sfermion soft terms.

input_type $=2 \quad$ sfermion soft terms given as absolute values of dimension mass ${ }^{2}$.

2. SUSY_FLAVOR uses the $W$ boson mass as a basic parameter rather than Fermi constant $G_{F}$. Therefore, in susy_flavor.in we use entry 30 of Block SMINPUTS (not used in the standard SLHA2) to define $M_{W}$.

3. We allow complex values for $\mu$ and two of the gaugino masses - chosen to be the $U(1)$ and $S U(2)$ mass terms $M_{1}$ and $M_{2}$. Their real and imaginary parts are defined in blocks EXTPAR and IMEXTPAR. We use $\tan \beta$ and the CP-odd Higgs mass $M_{A}$ as the input parameters for the Higgs sector.

4. Following the SLHA2 convention, we only define the upper triangle of each of the hermitian sfermion soft mass matrices in the MSL2IN, MSE2IN, MSQ2IN, MSD2IN, MSU2IN 
and IMMSL2IN, IMMSE2IN, IMMSQ2IN, IMMSD2IN, IMMSU2IN blocks. It is obligatory to define all entries, both diagonal and upper off-diagonal, since SUSY_FLAVOR does not read diagonal sfermion masses from the EXTPAR block. The iconv parameter defined in the SOFTINP block determines if sfermion parameters are given in SLHA2 or Ref. [14] conventions (see Table 2). Finally, the input_type parameter in the SOFTINP block defines the format of the off-diagonal mass terms. If input_type $=1$, the off-diagonal entries given in susy_flavor.in are assumed to be dimensionless mass insertions and the flavor violating sfermion mass terms are calculated as

$$
\left(m_{X}^{2}\right)_{I J}=\left(m_{X}^{2}\right)_{J I}^{\star}=\delta_{X}^{I J} \sqrt{\left(m_{X}^{2}\right)_{I I}\left(m_{X}^{2}\right)_{J J}}
$$

where $X=L, E, Q, U, D$ and $I, J$ enumerate superpartners of the mass-eigenstate quarks.

5. The blocks TEIN, TDIN, TUIN and IMTEIN, IMTDIN, IMTUIN define the trilinear sfermion mixing matrices which are generally non-hermitian. One is required to define all entries. As for the soft mass terms, the iconv parameter chooses the input convention, SLHA2 or Ref. [14]. For the trilinear mixing, the parameter input_type defines the format and dimension of both the diagonal and off-diagonal terms. If input_type $=1$, then all relevant susy_flavor.in entries are treated as dimensionless numbers and expanded to full trilinear mixing matrices using eqs. (25]26). For the diagonal LR terms, SUSY_FLAVOR uses the formulae

$$
\begin{aligned}
& A_{l}^{I I}=Y_{l}^{I}\left(\left(m_{L}^{2}\right)_{I I}\left(m_{E}^{2}\right)_{I I}\right)^{1 / 4} a_{l}^{I}, \\
& A_{d}^{I I}=Y_{d}^{I}\left(\left(m_{Q}^{2}\right)_{I I}\left(m_{D}^{2}\right)_{I I}\right)^{1 / 4} a_{d}^{I}, \\
& A_{u}^{I I}=Y_{u}^{I}\left(\left(m_{Q}^{2}\right)_{I I}\left(m_{U}^{2}\right)_{I I}\right)^{1 / 4} a_{u}^{I},
\end{aligned}
$$

where $a_{l}^{I}, a_{d}^{I}, a_{u}^{I}$ are the diagonal trilinear mixing terms read from the input file.

For the off-diagonal LR terms, SUSY_FLAVOR uses

$$
\begin{aligned}
& A_{l}^{I J}=\delta_{L L R}^{I J} \sqrt{2} / v_{1} \sqrt{\left(m_{L}^{2}\right)_{I I}\left(m_{E}^{2}\right)_{J J}}, \\
& A_{d}^{I J}=\delta_{D L R}^{I J} \sqrt{2} / v_{1} \sqrt{\left(m_{Q}^{2}\right)_{I I}\left(m_{D}^{2}\right)_{J J}} \\
& A_{u}^{I J}=\delta_{U L R}^{I J} \sqrt{2} / v_{2} \sqrt{\left(m_{Q}^{2}\right)_{I I}\left(m_{U}^{2}\right)_{J J}} .
\end{aligned}
$$

Note that in eqs. (25,26) for simplicity we use $\left(m_{Q}^{2}\right)_{I I}$ as the diagonal mass scale for both up and down left squark fields (in general related by the CKM rotation, see eqs. (20,22) ).

\subsection{Parameter initialization inside the program}

SUSY_FLAVOR input parameters can also be initialized directly inside the driver program using the set of routines described below. Before the proper initialization sequence, the user can set the iconv variable value to choose the input convention: 


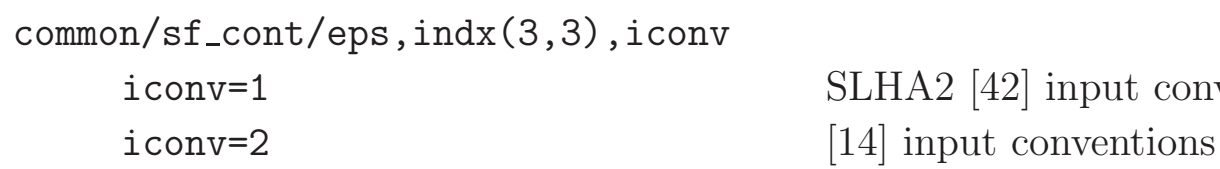

After choosing the input conventions, one should subsequently initialize the gauge, matter fermion, Higgs, SUSY fermion and sfermion sectors, using the procedures described in detail in the following sections.

\subsubsection{Gauge sector}

As input, SUSY_FLAVOR takes the gauge boson masses $\left(M_{W}, M_{Z}\right)$ and the gauge coupling constants (electromagnetic and strong) at the $M_{Z}$ scale. They are initialized by:

Routine and arguments

vpar_update (zm,wm, alpha_em)

$\mathrm{Zm}$

wm

alpha_em

lam_fit(alpha_s)

lam_fit_nlo(alpha_s)

alpha_s
Purpose and MSSM parameters

Sets electromagnetic sector parameters

$M_{Z}, Z$ boson mass

$M_{W}, W$ boson mass

$\alpha_{e m}\left(M_{Z}\right)$, QED coupling at $M_{Z}$ scale

Sets $\alpha_{s}\left(M_{Z}\right)$ and $\Lambda_{Q C D}$ for 4-6 flavors at the NNLO level

Sets $\alpha_{s}\left(M_{Z}\right)$ and $\Lambda_{Q C D}$ for $4-6$ flavors at the NLO level

$\alpha_{s}\left(M_{Z}\right)$, strong coupling at $M_{Z}$ scale

\subsubsection{Matter fermion sector}

SUSY_FLAVOR assumes that neutrinos are massless. Pole masses of the charged leptons are initialized in the file mh_init.f in block data init_phys. They are stored in the em array in common/fmass/em(3), um(3), dm(3) and can be directly modified there. Their default values are:

$\begin{array}{ll}\text { Lepton mass } & \text { Value } \\ m_{e} & \mathrm{em}(1)=0.000511 \\ m_{\mu} & \mathrm{em}(2)=0.105659 \\ m_{\tau} & \mathrm{em}(3)=1.777\end{array}$

In the quark sector the most important input parameters are the running top and bottom masses at a given renormalization scale and the CKM matrix angles and phase. They can be set by: 
Routine and arguments

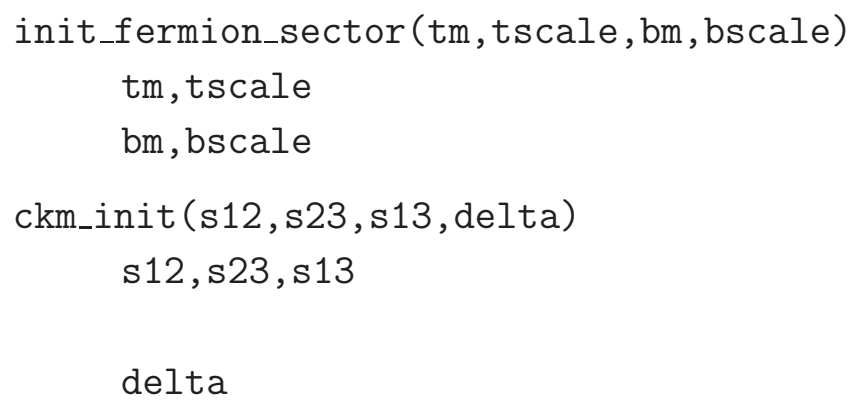

Purpose and MSSM parameters

Sets running top and bottom quark mass $m_{t}\left(\mu_{t}\right)$, running $\overline{\mathrm{MS}}$ top quark mass $m_{b}\left(\mu_{b}\right)$, running $\overline{\mathrm{MS}}$ bottom quark mass

Initialization of the CKM matrix $\sin \theta_{12}, \sin \theta_{23}, \sin \theta_{13}$, sines of the CKM angles

$\delta$, the CKM phase in radians

The light quark masses are also initialized in the block data init_phys of the file mh_init.f and stored in common/fmass_high/umu (3) , uml (3), amuu (3), dmu (3), dml (3), amud (3). The arrays uml(dml) contain up(down) quark masses at the scale amuu(amud), respectively. Their default values are:

$\begin{array}{lll}\text { Running quark mass } & \text { Mass value } & \text { Mass scale } \\ m_{d}\left(\mu_{d}\right) & \operatorname{dml}(1)=0.007 & \operatorname{amud}(1)=2 \\ m_{s}\left(\mu_{s}\right) & \operatorname{dml}(2)=0.11 & \operatorname{amud}(2)=2 \\ m_{b}\left(\mu_{b}\right) & \operatorname{dml}(3)=4.17 & \operatorname{amud}(3)=4.17 \\ m_{u}\left(\mu_{u}\right) & \operatorname{uml}(1)=0.004 & \operatorname{amuu}(1)=2 \\ m_{c}\left(\mu_{c}\right) & \operatorname{uml}(2)=1.279 & \operatorname{amuu}(2)=1.279 \\ m_{t}\left(\mu_{t}\right) & \operatorname{uml}(3)=163.5 & \operatorname{amuu(3)}=163.5\end{array}$

The variables of the arrays uml, amuu, dml, amud can be directly accessed and modified if necessary. However, for consistency, after such modifications the user should call the routine init_run_qmass which calculates running quark masses at the high $m_{t}$ scale (stored in common/fmass_high/ in the arrays umu,dmu and in common/fmass/ in the arrays um,dm) for later use in the running Yukawa couplings and in SUSY loop calculations.

\subsubsection{Higgs sector}

Following the common convention, we take the Higgs mixing parameter $\mu$, the CP-odd Higgs boson mass $M_{A}$, and the ratio of vacuum expectation values $\tan \beta=v_{2} / v_{1}$ as the input parameters. Other Higgs sector parameters listed in Section 2.1 can be expressed as:

$$
\begin{aligned}
m_{H_{1}}^{2} & =\frac{1}{2}\left(M_{A}^{2}-2|\mu|^{2}-\left(M_{A}^{2}+M_{Z}^{2}\right) \cos 2 \beta\right) \\
m_{H_{2}}^{2} & =\frac{1}{2}\left(M_{A}^{2}-2|\mu|^{2}+\left(M_{A}^{2}+M_{Z}^{2}\right) \cos 2 \beta\right) \\
m_{12}^{2} & =-\frac{M_{A}^{2}}{2 \sin 2 \beta} .
\end{aligned}
$$

The MSSM Higgs sector at the tree level can be effectively parametrized in terms of just $M_{A}$ and $\tan \beta$, but the $\mu$ parameter is necessary for the chargino and neutralino sectors. Here it 
is used to calculate the original Higgs soft mass parameters $m_{H_{1}}^{2}$ and $m_{H_{2}}^{2}$ for completeness and future applications; they currently have no further use.

$\begin{array}{cl}\begin{array}{cl}\text { Routine and arguments } \\ \text { init_higgs_sector }(\mathrm{pm}, \mathrm{tb}, \mathrm{amu}, \text { ierr) }\end{array} & \text { Purpose and MSSM parameters } \\ \mathrm{pm} & \text { Higgs sector initialization } \\ \mathrm{tb} & \text { CP-odd Higgs mass } M_{A} \\ \mathrm{amu} & \text { Ratio of Higgs VEVs, tan } \beta=\frac{v_{2}}{v_{1}} \\ \text { ierr } & \text { Higgs mixing parameter } \mu \text { (complex) } \\ & \text { output error code: ierr } \neq 0 \text { if Higgs sector } \\ & \text { initialization failed } \\ \text { init_yukawa } & \text { Initialization of the running Yukawa cou- } \\ & \text { plings } Y_{l}, Y_{u}, Y_{d} \text { for all generations (at the } \\ & \text { same scale as the running quark masses) }\end{array}$

\subsubsection{Supersymmetric fermion sector}

Initialization is done by the routine:

Routine and arguments

init_ino_sector (gm1, gm2, gm3, amu, tb, ierr)

gm1, gm2

gm3

tb

amu

ierr
Purpose and MSSM parameters gaugino sector initialization $U(1), S U(2)$ gaugino masses (complex) $S U(3)$ gaugino mass $\tan \beta=\frac{v_{2}}{v_{1}}$, the ratio of Higgs VEVs the Higgs mixing parameter $\mu$ (complex) output warning code: ierr $\neq 0$ if a chargino or a neutralino is lighter than $M_{Z} / 2$

If one sets $M_{1}=0$ in the call to init_ino_sector then the GUT-derived relation $M_{1}=$ $\frac{5}{3} \tan ^{2} \theta_{W} M_{2}$ is used in the gaugino mass calculations.

\subsubsection{Sfermion sector}

This is the most complicated MSSM sector; it contains a large number of free parameters. SUSY_FLAVOR supplies two subroutines for the sfermion parameters initialization, init_slepton_sector and init_squark_sector. They accept as input only dimensionless mass insertions and dimensionless diagonal trilinear soft mixing terms, expanded in SUSY_FLAVOR to entries of the soft mass matrices as defined by eqs. (24,25]26) (this is only a particular choice of parametrization and does not lead to any loss of generality). The sfermion initialization routines have the following arguments: 


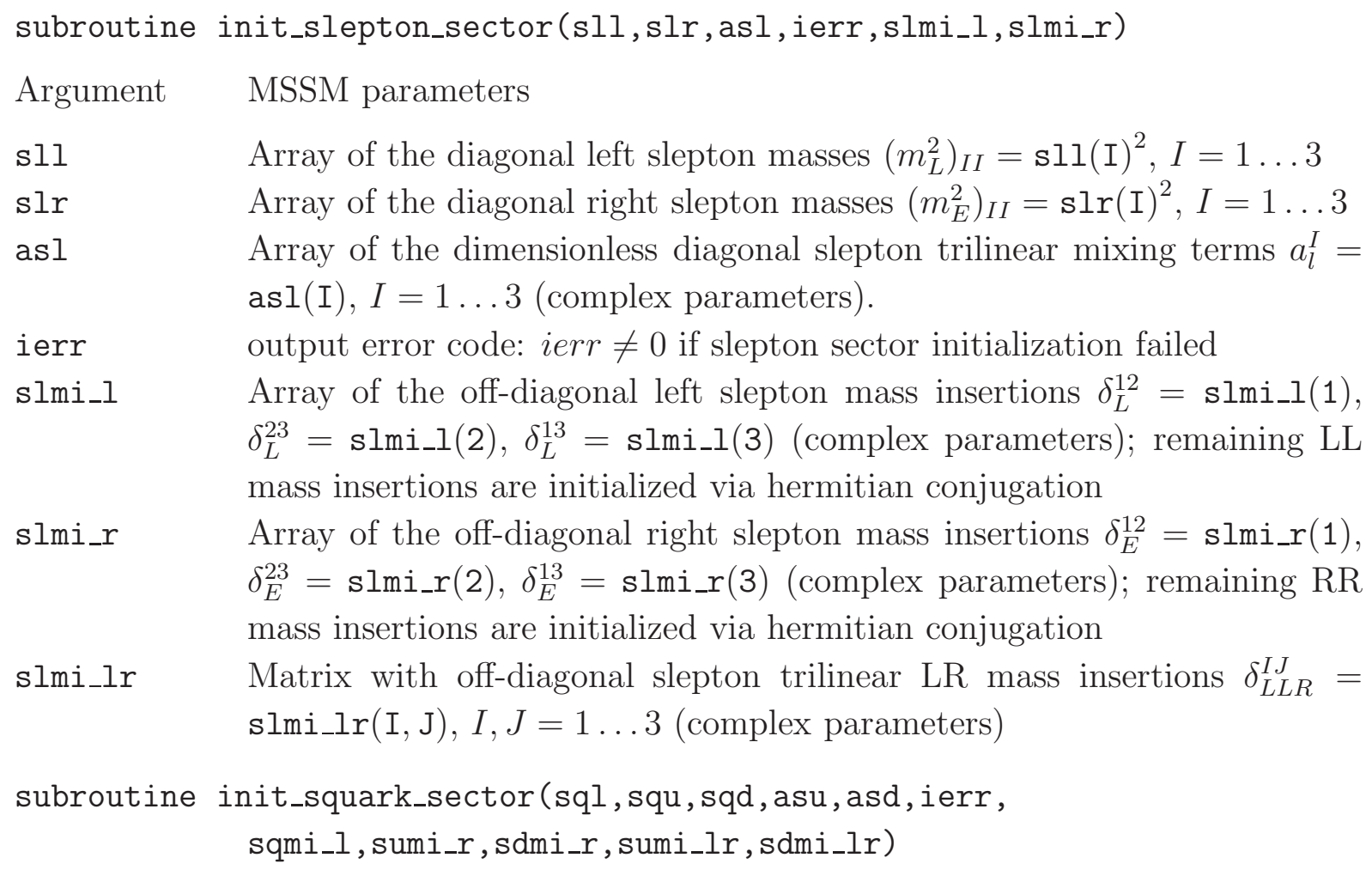
$\delta_{Q}^{23}=$ sqmi_l(2), $\delta_{Q}^{13}=$ sqmi_l(3) (complex parameters); remaining QLL mass insertions are initialized via hermitian conjugation

sumi_r Array of the off-diagonal right up-squark mass insertions $\delta_{U}^{12}=$ sumi_r(1), $\delta_{U}^{23}=$ sumi_r(2), $\delta_{U}^{13}=$ sumi_r(3) (complex parameters); remaining URR mass insertions are initialized via hermitian conjugation

sdmi_r Array of the off-diagonal right down-squark mass insertions $\delta_{D}^{12}=$ sdmi_r(1), $\delta_{D}^{23}=$ sdmi_r(2), $\delta_{D}^{13}=$ sdmi_r(3) (complex parameters); remaining DRR mass insertions are initialized via hermitian conjugation

sumi_lr Matrix with off-diagonal up-squark trilinear LR mass insertions $\delta_{U L R}^{I J}=$ sumi_lr(I, J), I, $J=1 \ldots 3$ (complex parameters)

sdmi_lr Matrix with off-diagonal down-squark trilinear LR mass insertions $\delta_{D L R}^{I J}=$ sdmi_lr(I, J), I, $J=1 \ldots 3$ (complex parameters) 
If necessary, experienced SUSY_FLAVOR users can directly modify the soft breaking sfermion parameters stored in common blocks /msoft/ and /soft/ (see Table团in Section 4.3). One must subsequently call the routines sldiag, sqdiag (see file mh_diag.f) to recalculate the tree-level sfermion masses and mixing matrices. This may, however, require a deeper understanding of the SUSY_FLAVOR initialization sequence and its data structure.

\subsection{Tree-level physical masses and mixing angles}

After performing the full initialization sequence in SUSY_FLAVOR, all the MSSM Lagrangian parameters listed in Section 2.1. physical tree-level particle masses (with the exception of the running quark masses), and mixing matrices are calculated and stored in common blocks. If necessary, they can be directly accessed and modified. Note, however, that after any modifications of the Lagrangian parameters, relevant procedures calculating physical masses and mixing angles have to called again. In Table 4 we list the important blocks storing MSSM parameters. Common blocks containing masses and mixing angles are listed in Table 5 ,

All parameters, tree-level masses, and mixing angles can be printed for test purposes, e.g. by calling the subroutines print_MSSM_par and print_MSSM_masses.

\section{$5 \quad$ List of processes}

In this section we list the set observables whose computation is fully implemented in SUSY_FLAVOR v1.0. For all of them, SUSY_FLAVOR takes into account one-loop supersymmetric contributions. QCD corrections and hadronic matrix elements are extracted from the papers of various authors, mostly from analyses done in the Standard Model. They are assumed to work reasonably well also in the MSSM since supersymmetric strong corrections from gluino and squarks are suppressed by large masses of these particles.

In most cases, QCD and hadronic corrections are known at the level of few to tens \%, while variations of supersymmetric flavor and $\mathrm{CP}$ violating parameters can change observables by orders-of-magnitude. Thus, as long as the MSSM parameters are not measured very precisely, the current implementation of strong corrections is sufficient for analyses performed in the framework of the general MSSM.

Calculations of the hadronic matrix elements are particularly difficult as they have to be performed, at least partially, in the regime of strongly coupled QCD. Results of such calculations can differ significantly depending on the methods used and thus carry significant theoretical uncertainties. Therefore, in SUSY_FLAVOR, quantities which requires hadronic 
Common block and variables

Lagrangian parameters

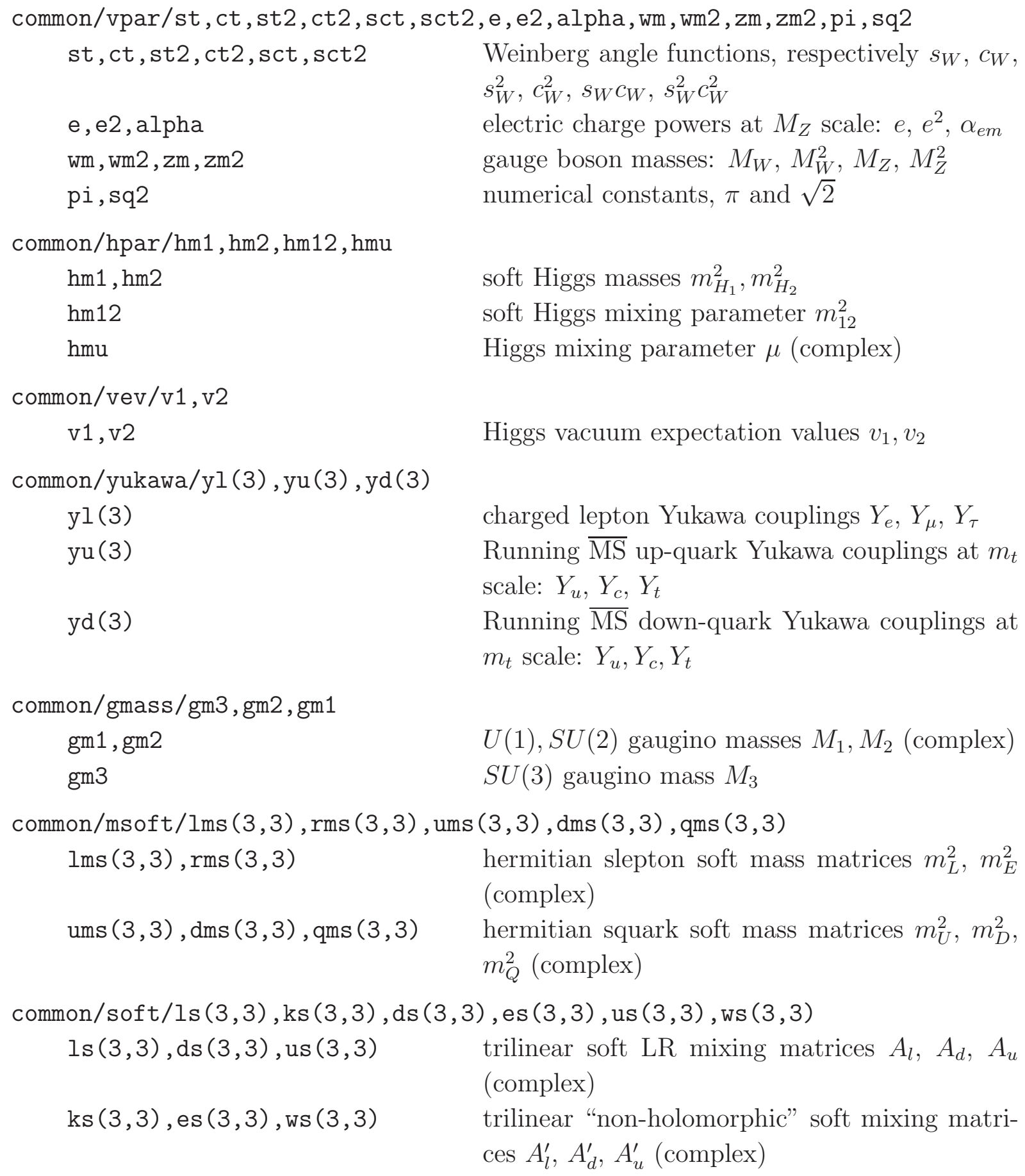

Table 4: Common blocks storing the MSSM Lagrangian parameters. We omit flavor indices in the fermion and sfermion sectors. 


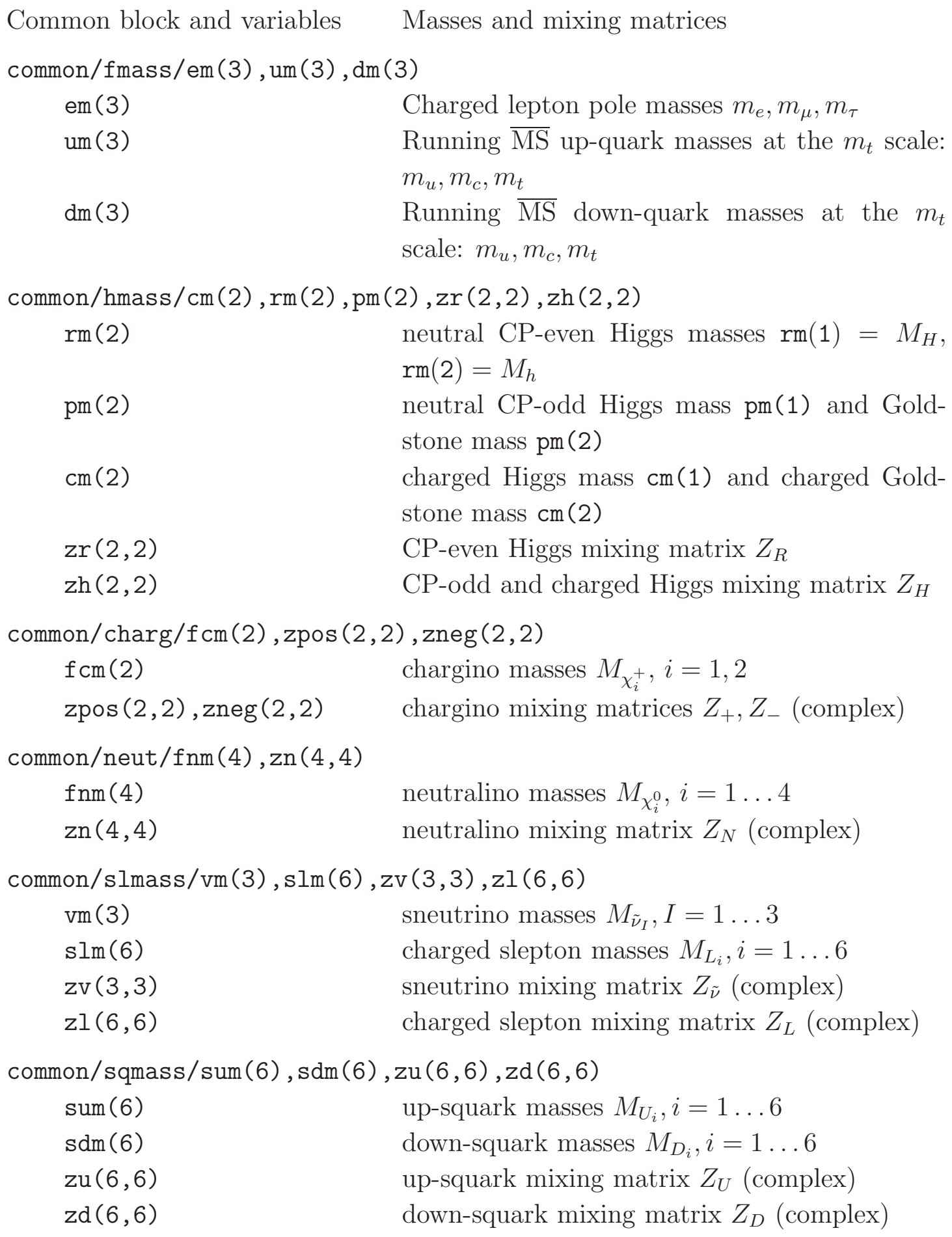

Table 5: Common blocks storing physical particle masses and mixing matrices. 
matrix element estimates and other QCD related quantities are treated as external parameters. They are initialized to the default values listed below for each observable and can be directly modified by users by changing the relevant variables in the common blocks where they are stored. Currently most of the hadronic (and related) input parameters used in SUSY_FLAVOR are taken from the Table 3 of Ref. [46].

\subsection{Electric Dipole Moments of charged leptons}

Lepton EDMs are defined as the coefficient $d_{l^{I}}$ in the effective Hamiltonian for the flavordiagonal lepton-lepton-photon interaction:

$$
\mathcal{H}_{e}=\frac{i d_{l^{I}}}{2} \bar{l}^{I} \sigma_{\mu \nu} \gamma_{5} l^{I} F^{\mu \nu}
$$

where $I=1,2,3$ is the generation index of the lepton as usual. In SUSY_FLAVOR lepton EDM is calculated by:

Routine: double precision function edm_l(I)

Input: $\quad I=1,2,3$ for $e, \mu, \tau$ respectively

Output: $\quad$ EDM for the charged lepton specified by $I$

QCD related factors: none, QCD corrections are small and not included

Details of calculations: Ref. [4]

\subsection{Neutron Electric Dipole Moment}

The neutron EDM can be approximated by the sum of the electric dipole moments of the constituent $d$ and $u$ quarks plus contributions of the chromoelectric dipole moments (CDM) of quarks and gluons. The EDMs of the individual quarks are defined analogously to eq. (28). The $\mathrm{CDM} c_{q}$ of quark $q$ is defined as:

$$
\mathcal{H}_{c}=-\frac{i c_{q}}{2} \bar{q} \sigma_{\mu \nu} \gamma_{5} T^{a} q G^{\mu \nu a}
$$

The gluonic dipole moment $c_{g}$ is defined as:

$$
\mathcal{H}_{g}=-\frac{c_{g}}{6} f_{a b c} G_{\mu \rho}^{a} G_{\nu}^{b \rho} G_{\lambda \sigma}^{c} \epsilon^{\mu \nu \lambda \sigma} .
$$

The exact calculation of the neutron EDM requires knowledge of its hadronic wave function. SUSY_FLAVOR uses the "naïve" chiral quark model approximation [47]:

$$
E_{n}=\frac{\eta_{e}}{3}\left(4 d_{d}-d_{u}\right)+\frac{e \eta_{c}}{4 \pi}\left(4 c_{d}-c_{u}\right)+\frac{e \eta_{g} \Lambda_{X}}{4 \pi} c_{g}
$$

where $\eta_{i}$ and $\Lambda_{X}$ are the QCD correction factors [48] and the chiral symmetry breaking scale [47, respectively. Various models give significantly different $\eta_{i}$ factors. As a result, even the sign of the neutron EDM is not certain. Thus the SUSY_FLAVOR result should be 
treated as an order of magnitude estimate only. The calculations are performed by calling

Routine

Input

Output

QCD related factors:

common/edm_qcd/eta_e, eta_c, eta_g, alamx

$\eta_{e}$

$\eta_{g}$

$\eta_{g}$

$\Lambda_{X}$ double precision function edm_n()

none

neutron EDM

Details of calculations: Ref. 4

eta_e $=1.53$

eta_c $=3.4$

eta_g $=3.4$

alamx $=1.18$

\section{3 $K_{L}^{0} \rightarrow \pi^{0} \bar{\nu} \nu$ and $K^{+} \rightarrow \pi^{+} \bar{\nu} \nu$ decay rates}

The relevant part of the effective Hamiltonian generated by the top quark and SUSY particle exchanges can be written as

$$
\mathcal{H}_{\mathrm{eff}}=\frac{G_{\mathrm{F}}}{\sqrt{2}} \frac{\alpha}{2 \pi \sin ^{2} \theta_{\mathrm{w}}} \sum_{l=e, \mu, \tau}\left[X_{L}(\bar{s} d)_{V-A}\left(\bar{\nu}_{l} \nu_{l}\right)_{V-A}+X_{R}(\bar{s} d)_{V+A}\left(\bar{\nu}_{l} \nu_{l}\right)_{V-A}\right] .
$$

The branching ratios for the $K \rightarrow \pi \nu \bar{\nu}$ decays are then given by

$$
\begin{aligned}
B r\left(K^{+} \rightarrow \pi^{+} \bar{\nu} \nu\right)=\kappa_{+} & {\left[\left(\frac{\Im \mathrm{m}\left(X_{L}+X_{R}\right)}{\lambda^{5}}\right)^{2}+\left(\frac{\Re \mathrm{e}\left(K_{c s}^{\star} K_{c d}\right)}{\lambda} P_{c}+\frac{\Re \mathrm{e}\left(X_{L}+X_{R}\right)}{\lambda^{5}}\right)^{2}\right] } \\
& \operatorname{Br}\left(K_{L}^{0} \rightarrow \pi^{0} \bar{\nu} \nu\right)=\kappa_{L}\left(\frac{\Im \mathrm{m}\left(X_{L}+X_{R}\right)}{\lambda^{5}}\right)^{2}
\end{aligned}
$$

where $\kappa$ [49], $\lambda$ (one of the Wolfenstein parameters [50]), and the NLO charm quark contribution $P_{c}$ [45, 51,52, can be modified by SUSY_FLAVOR users (note that $\kappa$ and $P_{c}$ depend on $V_{u s}, m_{c}$ and $\alpha_{s}$ ) Branching ratio calculations are performed by calling

Routine subroutine k_pivv(br_k0,br_kp)

Input none

Output $\quad$ br_k0 $=\operatorname{Br}\left(K_{L}^{0} \rightarrow \pi^{0} \bar{\nu} \nu\right)$

QCD related factors

$$
\mathrm{br \_ kp}=\operatorname{Br}\left(K^{+} \rightarrow \pi^{+} \bar{\nu} \nu\right)
$$

common/kpivv/ak0, del_ak0, akp, del_akp, pc, del_pc, alam

$$
\begin{array}{ll}
\kappa_{L} \pm \Delta \kappa_{L} & \text { ak0 }=2.231 \cdot 10^{-10}, \text { del_ak0 }=0.013 \cdot 10^{-10} \\
\kappa_{+} \pm \Delta \kappa_{+} & \text {akp }=5.173 \cdot 10^{-11}, \text { del_akp }=0.025 \cdot 10^{-11} \\
P_{c} \pm \Delta P_{c} & \text { pc }=0.41, \text { del_pc }=0.03 \\
\lambda & \text { alam }=0.225
\end{array}
$$

Details of calculations: $\quad$ Ref. [11] 


\section{$5.4 \quad B_{d}^{0} \rightarrow l^{I+} l^{J-}$ and $B_{s}^{0} \rightarrow l^{I+} l^{J-}$ decay rates}

The general expression for these branching ratios are rather complicated and can be found in [12. For most users it is sufficient to know that, in addition to the MSSM parameters, the dilepton $B$ decays depend on the $B$ meson masses and the hadronic matrix elements of the down quark vector and scalar currents:

$$
\begin{aligned}
\left\langle 0\left|\bar{b} \gamma_{\mu} P_{L(R)} s\right| B_{s(d)}(p)\right\rangle & =-(+) \frac{i}{2} p_{\mu} f_{B_{s(d)}} \\
\left\langle 0\left|\bar{b} P_{L(R)} s\right| B_{s(d)}(p)\right\rangle & =+(-) \frac{i}{2} \frac{M_{B_{s(d)}}^{2} f_{B_{s}}}{m_{b}+m_{s(d)}}
\end{aligned}
$$

where $p_{\mu}$ is the momentum of the decaying $B_{s(d)}$-meson of mass $M_{B_{s(d)}}$. The $B_{d}^{0} \rightarrow l^{I+} l^{J-}$ and $B_{s}^{0} \rightarrow l^{I+} l^{J-}$ decay branching ratios are calculated by:

Routine double precision function $\mathrm{b}_{-} \mathrm{ll}(\mathrm{K}, \mathrm{L}, \mathrm{I}, \mathrm{J})$

Input $\quad I, J=1,2,3$ - outgoing leptons generation indices

$K, L$ - generation indices of the valence quarks of the $B^{0}$ meson: setting $(K, L)=(3,1),(1,3),(3,2)$ and $(2,3)$ chooses respectively $B_{d}^{0}, \bar{B}_{d}^{0}, B_{s}^{0}$ and $\bar{B}_{s}^{0}$ decay

Output Branching ratios of the decay defined by $K, L, I, J$

QCD related factors

common/meson_data/dmk, amk, epsk, fk, dmd, amd, fd, amb (2), dmb (2), gam_b(2),fb(2)

$M_{B_{d}}$

$M_{B_{s}}$

$f_{B_{d}}$

$f_{B_{s}}$

Details of calculations:

$$
\begin{aligned}
& \operatorname{amb}(1)=5.2794 \\
& \operatorname{amb}(2)=5.368 \\
& \mathrm{fb}(1)=0.2 \\
& \mathrm{fb}(2)=0.245
\end{aligned}
$$

\section{$5.5 \bar{K}^{0} K^{0}$ meson mixing parameters}

SUSY_FLAVOR calculates two parameters measuring the amount of CP-violation in neutral $K$ meson oscillations: $\varepsilon_{K}$ and the $\bar{K}^{0}-K^{0}$ mass difference $\Delta M_{K}$.

$$
\begin{gathered}
\Delta M_{K}=2 \Re \mathrm{e}\left\langle\bar{K}^{0}\left|H_{\mathrm{eff}}^{\Delta S=2}\right| K^{0}\right\rangle, \\
\varepsilon_{K}=\frac{\exp (i \pi / 4)}{\sqrt{2} \Delta M_{K}} \Im \mathrm{m}\left\langle\bar{K}^{0}\left|H_{\mathrm{eff}}^{\Delta S=2}\right| K^{0}\right\rangle .
\end{gathered}
$$

QCD dependent corrections are known with reasonable accuracy for the $\varepsilon_{K}$ parameter. The long distance contributions to $\Delta M_{K}$ are large and difficult to control. Thus the result given by SUSY_FLAVOR for $\Delta M_{K}$ should be treated as an order of magnitude estimate only. 
Apart from the MSSM parameters, the calculation of the $\bar{K}^{0} K^{0}$ meson mixing requires knowledge of the meson masses and of the hadronic matrix elements of the following set of four-quark operators:

$$
\begin{aligned}
Q_{1}^{\mathrm{VLL}} & =\left(\bar{q}_{\alpha}^{I} \gamma_{\mu} P_{L} q_{\alpha}^{J}\right)\left(\bar{q}_{\beta}^{I} \gamma^{\mu} P_{L} q_{\beta}^{J}\right), \\
Q_{1}^{\mathrm{LR}} & =\left(\bar{q}_{\alpha}^{I} \gamma_{\mu} P_{L} q_{\alpha}^{J}\right)\left(\bar{q}_{\beta}^{I} \gamma^{\mu} P_{R} q_{\beta}^{J}\right), \\
Q_{2}^{\mathrm{LR}} & =\left(\bar{q}_{\alpha}^{I} P_{L} q_{\alpha}^{J}\right)\left(\bar{q}_{\beta}^{I} P_{R} q_{\beta}^{J}\right), \\
Q_{1}^{\mathrm{SLL}} & =\left(\bar{q}_{\alpha}^{I} P_{L} q_{\alpha}^{J}\right)\left(\bar{q}_{\beta}^{I} P_{L} q_{\beta}^{J}\right), \\
Q_{2}^{\mathrm{SLL}} & =\left(\bar{q}_{\alpha}^{I} \sigma_{\mu \nu} P_{L} q_{\alpha}^{J}\right)\left(\bar{q}_{\beta}^{i} \sigma^{\mu \nu} P_{L} q_{\beta}^{J}\right)
\end{aligned}
$$

where $\alpha, \beta$ are color indices, for the $\bar{K}^{0} K^{0}$ mixing one should choose flavor indices $I=2$ and $J=1$. The matrix elements can be written as:

$$
\begin{aligned}
\left\langle\bar{K}^{0}\left|Q_{1}^{\mathrm{VLL}}(\mu)\right| K^{0}\right\rangle & =\frac{1}{3} M_{K} F_{K}^{2} B_{1}^{\mathrm{VLL}}(\mu), \\
\left\langle\bar{K}^{0}\left|Q_{1}^{\mathrm{LR}}(\mu)\right| K^{0}\right\rangle & =-\frac{1}{6}\left(\frac{M_{K}}{m_{s}(\mu)+m_{d}(\mu)}\right)^{2} M_{K} F_{K}^{2} B_{1}^{\mathrm{LR}}(\mu), \\
\left\langle\bar{K}^{0}\left|Q_{2}^{\mathrm{LR}}(\mu)\right| K^{0}\right\rangle & =\frac{1}{4}\left(\frac{M_{K}}{m_{s}(\mu)+m_{d}(\mu)}\right)^{2} M_{K} F_{K}^{2} B_{2}^{\mathrm{LR}}(\mu), \\
\left\langle\bar{K}^{0}\left|Q_{1}^{\mathrm{SLL}}(\mu)\right| K^{0}\right\rangle & =-\frac{5}{24}\left(\frac{M_{K}}{m_{s}(\mu)+m_{d}(\mu)}\right)^{2} M_{K} F_{K}^{2} B_{1}^{\mathrm{SLL}}(\mu), \\
\left\langle\bar{K}^{0}\left|Q_{2}^{\mathrm{SLL}}(\mu)\right| K^{0}\right\rangle & =-\frac{1}{2}\left(\frac{M_{K}}{m_{s}(\mu)+m_{d}(\mu)}\right)^{2} M_{K} F_{K}^{2} B_{2}^{\mathrm{SLL}}(\mu),
\end{aligned}
$$

where $F_{K}$ is the $K$-meson decay constant. By default, SUSY_FLAVOR uses the $B_{i}^{X}$ values at the scale $\mu=2 \mathrm{GeV}$ given in 53] using the NDR renormalization scheme (quark masses at the scale $2 \mathrm{GeV}$ are stored in common/fmass_high/, see Section 4.2.2).

In addition to the hadronic matrix elements, QCD corrections depend also on the " $\eta$ " factors describing the evolution of the relevant Wilson coefficients from the high to low energy scale. These factors are automatically calculated at NLO by SUSY_FLAVOR. For the SM contribution to the Wilson coefficient of the $Q^{\mathrm{VLL}}$ operator a separate careful calculation of the evolution factors has been performed [54,55]. Therefore SUSY_FLAVOR treats this contribution separately, setting $B_{S M}^{\mathrm{VLL}}$ and the $\eta_{S M}$ factor to default values given in [56] (see [53] for a very detailed discussion of the structure of the QCD corrections in $\bar{B}^{0} B^{0}$ and $\bar{K}^{0} K^{0}$ systems, including their renormalization scheme dependence and calculations of the evolution factors " $\eta$ " implemented in SUSY_FLAVOR).

The kaon mass difference $\Delta M_{K}$ and the $\varepsilon_{K}$ parameter measuring the amount of CP violation in $\bar{K}^{0} K^{0}$ mixing are calculated by 


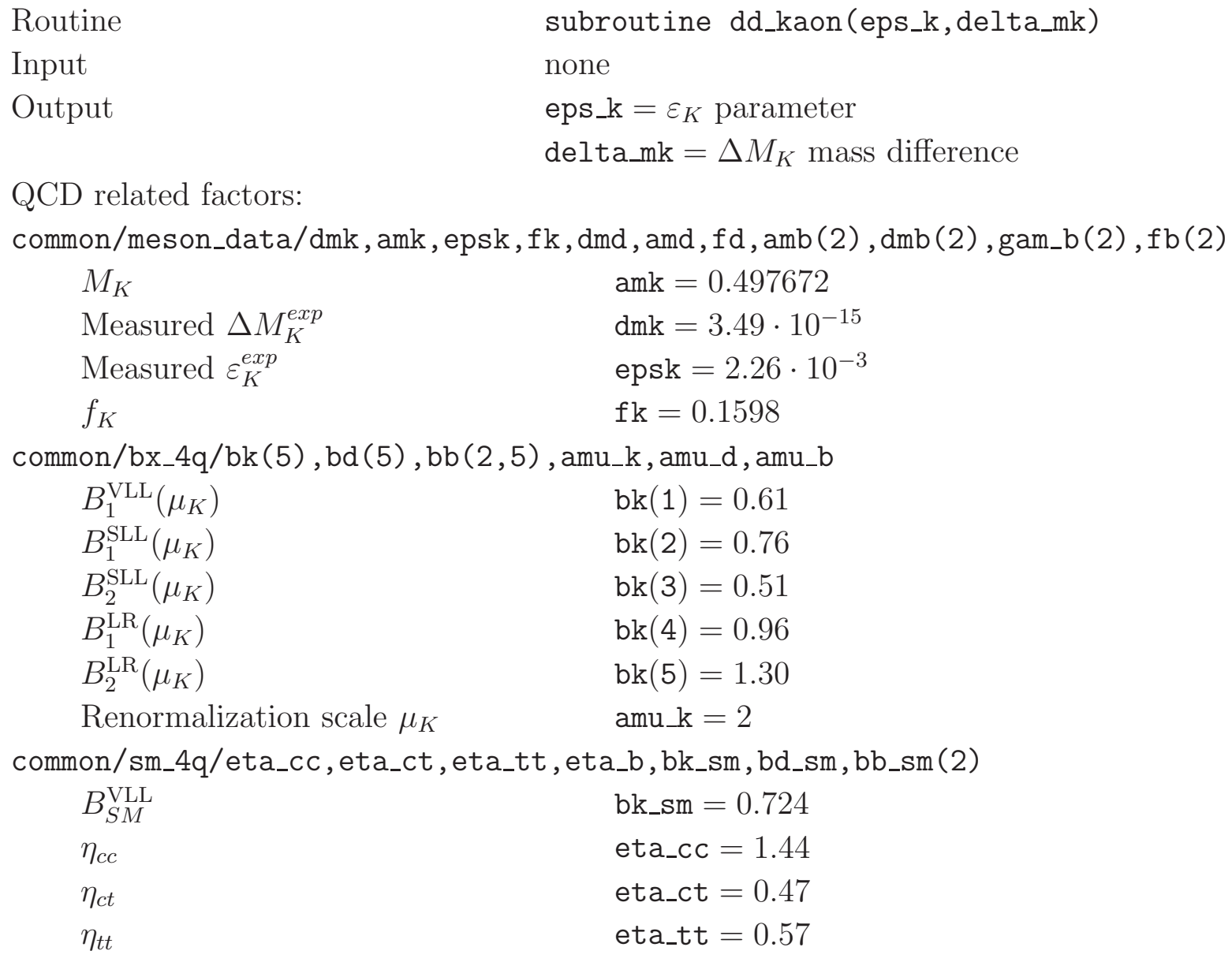

Details of calculations: $\quad$ Ref. [10, 53]

\section{$5.6 \quad \bar{D}^{0} D^{0}$ meson mass difference}

Calculations of the mass difference $\Delta m_{D}$ of the neutral $D$ mesons have large theoretical uncertainties due to unknown long-distance strong corrections. Thus, as in the case of $\Delta m_{K}$, the SUSY_FLAVOR result for $\Delta m_{D}$ should be treated as an order of magnitude estimate only.

The structure of strong corrections is analogous to those in the $K$ meson system. However, in this case hadronic matrix elements and QCD evolution calculations available in the literature are much less refined. SUSY_FLAVOR uses the NLO evolution for the " $\eta$ " factors and sets, by default, all the relevant hadronic matrix elements $B_{i}=1$, i.e. it uses the "vacuum saturation" approximation (this can be changed easily when new results become available). 


\begin{tabular}{|c|c|}
\hline Routine & subroutine uu_bmeson(delta_md) \\
\hline Input & none \\
\hline Output & delta_md $=\Delta M_{D}$ mass difference \\
\hline \multicolumn{2}{|l|}{ QCD related factors: } \\
\hline \multicolumn{2}{|c|}{ common/meson_data/dmk, amk, epsk, fk, dmd, amd, fd, amb (2), dmb (2), gam_b (2), fb (2) } \\
\hline$M_{D}$ & amd $=1.8645$ \\
\hline Measured $\Delta M_{D}^{\exp }$ & $\mathrm{dmd}=4.61 \cdot 10^{-14}$ \\
\hline$f_{D}$ & $\mathrm{fd}=0.165$ \\
\hline \multicolumn{2}{|c|}{ common/bx_4q/bk (5), bd (5), bb $(2,5)$, amu_k, amu_d, amu_b } \\
\hline$B_{1}^{\mathrm{VLL}}\left(\mu_{D}\right)$ & $\operatorname{bd}(1)=1$ \\
\hline$B_{1}^{\mathrm{SLL}}\left(\mu_{D}\right)$ & $\mathrm{bd}(2)=1$ \\
\hline$B_{2}^{\mathrm{SLL}}\left(\mu_{D}\right)$ & $\mathrm{bd}(3)=1$ \\
\hline$B_{1}^{\mathrm{LR}}\left(\mu_{D}\right)$ & $\mathrm{bd}(4)=1$ \\
\hline$B_{2}^{\mathrm{LR}}\left(\mu_{D}\right)$ & $\operatorname{bd}(5)=1$ \\
\hline Renormalization scale $\mu_{D}$ & amu_d $=2$ \\
\hline \multicolumn{2}{|c|}{ common/sm_4q/eta_cc, eta_ct, eta_tt, eta_b, bk_sm, bd_sm, bb_sm (2) } \\
\hline$B_{S M}^{\mathrm{VLL}}$ & bd_sm $=1$ \\
\hline Details of calculations: & Performed by authors, unpublished \\
\hline
\end{tabular}

\section{7 $\quad \bar{B}_{d}^{0} B_{d}^{0}$ and $\bar{B}_{s}^{0} B_{s}^{0}$ mass differences}

Mixing and $\mathrm{CP}$ violation phenomena are also observed in the neutral $B$ meson systems. In particular, the mass differences in the $\bar{B}_{d}^{0} B_{d}^{0}$ and $\bar{B}_{s}^{0} B_{s}^{0}$ oscillations have been measured,

$$
\Delta M_{B_{d(s)}}=2\left|\left\langle\bar{B}_{d(s)}^{0}\left|H_{\mathrm{eff}}^{\Delta B=2}\right| B_{d(s)}^{0}\right\rangle\right|
$$

In addition to the MSSM parameters, theoretical calculations of $\Delta m_{B_{d}}$ and $\Delta m_{B_{s}}$ depend, as for $K$ and $D$ oscillations, on the relevant hadronic matrix elements and QCD evolution factors. The formulae for $\bar{B}^{0} B^{0}$ mixing can be obtained by making the obvious replacements in the formulae presented in Section 5.5. Currently SUSY_FLAVOR uses the same set of $B_{i}$ factors for both the $B_{d}$ and $B_{s}$ sectors, but it leaves the possibility to distinguish between them in future, if necessary. For this one needs to independently initialize the arrays $\mathrm{bb}(1, \mathrm{i})$ ( $B_{d}$ meson hadronic matrix elements) and $\mathrm{bb}(2, \mathrm{i})$ ( $B_{s}$ meson hadronic matrix elements) stored in common/bx_4q/.

The values of the $B$ meson masses and coupling constants are the same as those listed in Section 5.4. SUSY_FLAVOR calculates the mass differences $\Delta M_{B_{d(s)}}$ as defined by eq. (41): 


\begin{tabular}{|c|c|}
\hline Routine & subroutine dd_bmeson(i,delta_mb) \\
\hline Input & $\begin{array}{l}i=1,2-\text { generation index of the lighter valence } \\
\text { the } B^{0} \text { meson, i.e. } i=2 \text { chooses } B_{s}^{0} \text { and } i=1 \text { cho }\end{array}$ \\
\hline Output & $\begin{array}{l}\text { delta_mb }=\Delta m_{B_{d}} \text { for } i=1 \\
\text { delta_mb }=\Delta m_{B_{s}} \text { for } i=2\end{array}$ \\
\hline CD related factors: & \\
\hline ommon/meson_data/dmk, amk, & $f k, d m d, a m d, f d, a m b(2), d m b(2), g a m_{-} b(2), f b(2)$ \\
\hline Measured $\Delta M_{B_{d}}^{\exp }$ & $\operatorname{dmb}(1)=3.01 \cdot 10^{-13}$ \\
\hline Measured $\Delta M_{B_{s}}^{e x p}$ & $\operatorname{dmb}(2)=1.2 \cdot 10^{-11}$ \\
\hline Measured width $\Gamma_{B_{d}}^{\exp }$ & gam_b $(1)=1.53 \cdot 10^{-12}$ \\
\hline Measured width $\Gamma_{B_{s}}^{e x p}$ & gam_b $(1)=1.466 \cdot 10^{-12}$ \\
\hline common/bx_4q/bk (5), bd (5), bl & ) , amu_k, amu_d, amu_b \\
\hline$B_{1}^{\mathrm{VLL}}\left(\mu_{B}\right)$ & $\mathrm{bb}(1,1)=\mathrm{bb}(2,1)=0.87$ \\
\hline$B_{1}^{\mathrm{SLL}}\left(\mu_{B}\right)$ & $\mathrm{bb}(1,2)=\mathrm{bb}(2,2)=0.8$ \\
\hline$B_{2}^{\mathrm{SLL}}\left(\mu_{B}\right)$ & $\mathrm{bb}(1,3)=\mathrm{bb}(2,3)=0.71$ \\
\hline$B_{1}^{\mathrm{LR}}\left(\mu_{B}\right)$ & $\mathrm{bb}(1,4)=\mathrm{bb}(2,4)=1.71$ \\
\hline$B_{2}^{\mathrm{LR}}\left(\mu_{B}\right)$ & $\mathrm{bb}(1,5)=\mathrm{bb}(2,5)=1.16$ \\
\hline Renormalization scale $\mu_{B}$ & amu_b $=4.6$ \\
\hline ommon/sm_4q/eta_cc, eta_ct, & , eta_b, bk_sm, bd_sm, bb_sm (2) \\
\hline$B_{S M B_{d}}^{\mathrm{VLL}}$ & bb_sm $(1)=1.22$ \\
\hline$B_{S M B_{s}}^{\mathrm{VLL}}$ & bb_sm $(2)=1.22$ \\
\hline$\eta_{b}$ & eta_b $=0.55$ \\
\hline
\end{tabular}

Details of calculations:

Ref. [10]

\section{$5.8 \quad B^{0} \rightarrow X_{s} \gamma$ decay rate}

Both the SUSY contributions and the QCD corrections to the calculation of the $B^{0} \rightarrow X_{s} \gamma$ decay rate are quite complex. Their implementation in SUSY_FLAVOR is based on the SUSY loop calculations performed by the authors (not published in a general form) and on the QCD evolution published in [57]. There are no user-accessible QCD factors apart from the arguments of the bxg_nl routine.

$\begin{array}{ll}\text { Routine } & \text { double precision function bxg_nl(del,amiu_b) } \\ \text { Input } & \text { del }- \text { relative photon energy infrared cutoff scale, } \\ & E_{\gamma} \geq(1-\operatorname{del}) E_{\gamma}^{\text {max }}, 0<\text { del }<1 \\ & \text { amiu_b }- \text { renormalization scale } \\ & B r\left(B \rightarrow X_{s} \gamma\right) . \\ \text { Output } & \text { General SUSY diagrams unpublished, QCD correc- } \\ \text { Details of calculations: } & \text { tions based on [57] }\end{array}$




\section{Summary and Outlook}

We have presented SUSY_FLAVOR, a tool for calculating the set of important FCNC and CPV observables in the general $R$-parity conserving MSSM. All implemented physical quantities (listed in Table 1) can be calculated simultaneously for a given set of MSSM parameters. The calculations of the SUSY particle spectrum and flavor mixing matrices are performed exactly, so the code can be used for a completely general pattern of soft SUSY flavor violating terms and complex phases, without restrictions on the size of sfermion mass insertions.

Besides complete routines for calculating the physical observables, SUSY_FLAVOR also provides an extensive library of parton-level Green's functions and Wilson coefficients of many effective quark and lepton operators (see Table 3). This set actually contains many more functions than are necessary to compute the quantities listed in Table 1. These intermediate building blocks can be used by SUSY_FLAVOR users to construct amplitudes for processes beyond those already fully implemented by dressing appropriate combinations of available form factors in QCD corrections and hadronic matrix elements, without repeating tedious one-loop SUSY calculations from scratch. For instance, the form factors implemented in SUSY_FLAVOR for the analysis of $B \rightarrow X_{s} \gamma$ and $B_{d(s)} \rightarrow l^{+} l^{-}$decays [3, 12, are sufficient to also calculate the $B \rightarrow K l^{+} l^{-}$decay rate.

SUSY_FLAVOR internally uses the conventions of Ref. [14, however in order to facilitate comparison with other programs that analyze various sectors of MSSM, we have implemented an option to input parameters in the SLHA2 format [42].

SUSY_FLAVOR has been written in FORTRAN 77 and runs fairly quickly; it is capable of producing a reasonably wide-range scan over the MSSM parameters within hours or days on a typical personal computer.

The SUSY_FLAVOR library is an open project. We want to gradually add more features in its future versions. In particular, we plan to:

- add more observables in the $B$-meson system, like the CP asymmetries in $\bar{B} B$ meson mixing and in $B \rightarrow X_{s} \gamma$ decay, as well as observables associated with $B \rightarrow \mathrm{Kl}^{+} \mathrm{l}^{-}$ decay.

- add observables for lepton flavor-violating processes like $\ell^{J} \rightarrow \ell^{I} \gamma, \ell^{J} \rightarrow \ell^{K} \ell^{L} \ell^{M}$, and for the lepton anomalous magnetic moments, $(g-2)_{I}$

- include quantities related to FCNCs in the top sector, like $t \rightarrow c X$ with $X=$ $\gamma, Z, g, H$, in order to probe the flavor violation in up-squark mass matrices that are (almost) unconstrained to this moment.

- implement full resummation of leading large $\tan \beta$ effects beyond the MFV scenario.

With the increasing accuracy of experimental data on flavor and $\mathrm{CP}$ violation in rare 
processes, it may eventually become possible to not only constrain the MSSM parameters, but also, if significant deviations from the SM predictions are found, to recover their actual values. For that multi-process analysis, such as the one performed by SUSY_FLAVOR, will be necessary. Therefore, we hope that SUSY_FLAVOR becomes an important tool that is useful not only to theorists working on MSSM but also to experimentalists fitting the MSSM onto forthcoming data from the Tevatron, LHC, and $B$-factories.

\section{Acknowledgments}

Authors would like to thank A. Buras, T. Ewerth, M. Misiak, C. Savoy, E. Slawianowska and S. Pokorski for collaboration in performing theoretical calculations used in SUSY_FLAVOR and for helping to check and debug some of its sections. We would also like to thank W. Altmannshofer, D. Guadagnoli and M. Wick for careful checking the $\operatorname{Br}\left(B \rightarrow X_{s} \gamma\right)$ code and reporting some inconsistencies.

This work is supported by the RTN European Programme, MRTN-CT-2006-035505 (HEPTOOLS, Tools and Precision Calculations for Physics Discoveries at Colliders). JR was also supported in part the Polish Ministry of Science and Higher Education Grant N N202 230337. J.R. and A.D. acknowledge partial support by the EU FP6 Marie Curie Research and Training Network "UniverseNet" (MRTN-CT-2006-035863). P.T. was supported by a Marshall Scholarship and a National Science Foundation Graduate Research Fellowship.

\section{A Installation of the program}

The installation and execution of SUSY_FLAVOR is very simple. On Unix or Linux systems, just follow these steps :

1. Download the code from http://www.fuw.edu.pl/susy_flavor and unpack it.

2. Change directory into susy_flavor.

3. Edit Makefile and change F77 = gfortran and FOPT $=-0$-fno-automatic -Wall into your compiler name and options, respectively.

4. Exit Makefile and type make (or gmake).

5. If everything go through the code will ask you whether to read the input file susy_flavor . in or to use the parameters defined inside the driver file.

6. To run the code from now on just type ./sflav.

The authors tested SUSY_FLAVOR on Linux machines. With few straightforward modifications the procedure describe above can be adapted to install program on other systems. 
The output of the program is displayed on the screen. In addition a file named mssm_data.txt is created. It contains information about the MSSM Lagrangian parameters and the treelevel mass spectrum corresponding to the input parameter set. A sample set of input parameters and corresponding SUSY_FLAVOR output are listed in the following appendices.

\section{B Example of the SUSY FLAVOR initialization sequence}

Below we present the contents of susy_flavor.f, the master driver file for the SUSY_FLAVOR library. The driver program illustrates the correct initialization sequence for all relevant MSSM parameters (see Section 4) and shows how to perform calls to the routines calculating physical observables (Section 5).

The driver file asks if the input parameters should be given directly inside the program or read from the default input file named susy_flavor.in (in this case skipping the values given in the program). Defining the input parameters in the separate file is probably more straightforward, but the ability to initialize parameters from within the program could be more useful for performing multi-dimensional scans over the MSSM parameter space.

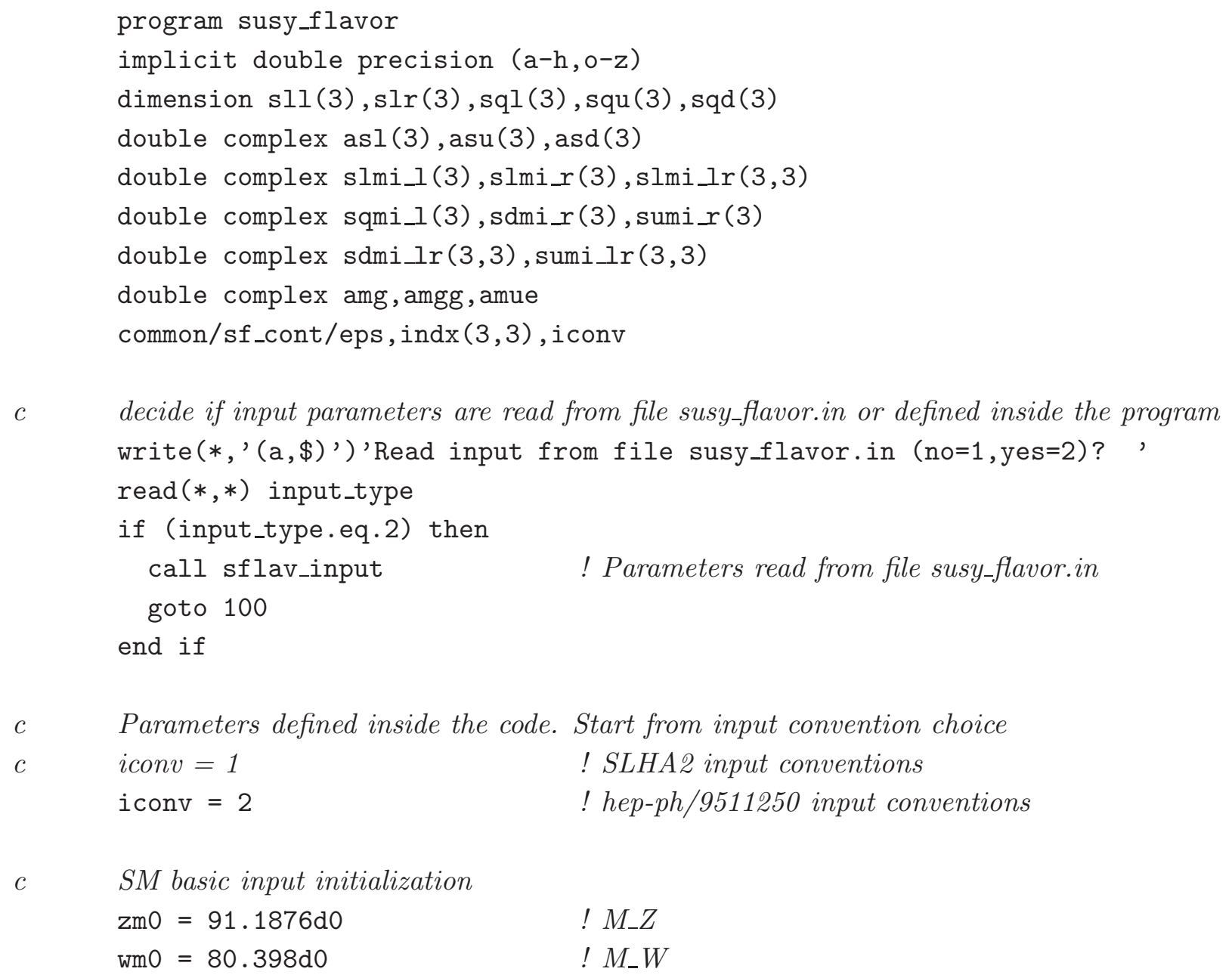


alpha_z $=1 / 127.934 \mathrm{~d} 0 \quad$ ! alpha_em(M_Z)

call vpar_update (zm0,wm0,alpha_z)

$c$

QCD parameters

alpha_s $=0.1172 \mathrm{~d} 0$

! alpha_s(M_Z)

call lam_fit(alpha_s)

! fits Lambda_QCD at 3 loop level

call lam_fit_nlo(alpha_s)

! fits Lambda_QCD at NLO level

$c$

CKM matrix initialization

$\begin{array}{ll}\text { alam }=0.2258 \mathrm{~d} 0 & \text { ! lambda } \\ \text { apar }=0.808 \mathrm{~d} 0 & ! \text { A } \\ \text { rhobar }=0.177 \mathrm{~d} 0 & \text { ! rho bar } \\ \text { etabar }=0.360 \mathrm{~d} 0 & \text { ! eta bar } \\ \text { call ckm_wolf (alam, apar,rhobar, etabar) }\end{array}$

c Fermion mass initialization, input: MSbar running quark masses

top_scale $=163.2 \mathrm{~d} 0$

top $=163.2 \mathrm{~d} 0 \quad ! m_{-} t($ top_scale $)$

bot_scale $=4.17 \mathrm{~d} 0$

bot $=4.17 \mathrm{~d} 0 \quad ! m_{-} b$ (bot_scale)

call init_fermion_sector(top, top_scale, bot, bot_scale)

c Higgs sector parameters

$\begin{array}{ll}\mathrm{pm}=200 & ! \text { M_A } \\ \text { tanbe }=10 & ! \text { tan(beta) } \\ \text { amue }=(200 . \mathrm{d} 0,100 . \mathrm{d} 0) & ! \text { mu parameter } \\ \text { call inithiggs_sector(pm,tanbe, amue, ierr) } \\ \text { if (ierr.ne.0) stop 'negative tree level Higgs mass }{ }^{2} ?\end{array}$

c Gaugino sector parameters: if $M 1=0$ set here then program uses $M 1=5 s_{W}^{2} / 3 c_{W}^{2}$ M2

amgg $=(0 . \mathrm{d} 0,0 . \mathrm{d} 0) \quad ! M 1$ (bino mass, complex)

amg $=(200 \cdot \mathrm{d} 0,0 . \mathrm{d} 0) \quad !$ M2 (wino mass, complex)

amglu $=3 * \operatorname{abs}(\mathrm{amg}) \quad !$ ! 3 (gluino mass)

call init_ino_sector (amgg, amg, amglu, amue, tanbe, ierr)

if (ierr.ne.0) write $(*, *)$ '-ino mass below M_Z/2?'

c Slepton diagonal soft breaking parameters
$\operatorname{sll}(1)=300 \cdot d 0$
! left selectron mass scale
$\operatorname{sll}(2)=300 \cdot d 0$
! left smuon mass scale
$\operatorname{sll}(3)=300 \cdot d 0$
! left stau mass scale
$\operatorname{slr}(1)=300 \cdot d 0$
! right selectron mass scale
$\operatorname{slr}(2)=300 \cdot d 0$
! right smuon mass scale
$\operatorname{slr}(3)=300 \cdot d 0$
! right stau mass scale
c Dimensionless (normalized to masses) slepton diagonal LR mixing 


$$
\begin{array}{ll}
\operatorname{asl}(1)=(1 \cdot \mathrm{d} 0,0 . \mathrm{d} 0) & \text { ! 1st generation } \\
\operatorname{asl}(2)=(1 \cdot \mathrm{d} 0,0 . \mathrm{d} 0) & \text { ! 2nd generation } \\
\operatorname{asl}(3)=(1 . \mathrm{d} 0,0 . \mathrm{d} 0) & \text { ! 3rd generation }
\end{array}
$$

$c \quad$ Slepton LL and $R R$ mass insertions (hermitian matrices, only upper part given)

$c \quad$ slmi_x(1),slmi_x(2), slmi_x(3) are 12,23,31 entry, respectively

$$
\begin{aligned}
& \text { do } i=1,3 \\
& \quad \operatorname{slmi} l(i)=(0 . \mathrm{d} 0,0 . \mathrm{d} 0) \quad \text { ! slepton LL mass insertion }
\end{aligned}
$$$$
\operatorname{slmi} r(i)=(0 . \mathrm{d} 0,0 . \mathrm{d} 0) \quad ! \text { slepton } R R \text { mass insertion }
$$

end do

slmi_l $(2)=(2 . \mathrm{d}-2,1 . \mathrm{d}-2) \quad$ ! example, non-vanishing LL 23 entry

$c \quad$ Slepton LR mass insertions, non-hermitian in general

do $i=1,3$

do $j=1,3$

slmi_lr $(i, j)=(0 . \mathrm{d} 0,0 . \mathrm{d} 0) !$ slepton LR ij mass insertion

end do

end do

c $\quad$ Calculate slepton physical masses and mixing angles

call init_slepton_sector(sll,slr,asl,ierr,slmi_l,slmi_r,slmi_lr)

if (ierr.ne.0) stop 'negative tree level slepton mass ${ }^{2}$ ?'

c $\quad$ Squark diagonal soft breaking parameters

$$
\begin{aligned}
& \mathrm{sql}(1)=500 \cdot \mathrm{d} 0 \quad \text { ! left squark mass, } 1 \text { st generation } \\
& \mathrm{sql}(2)=500 \cdot \mathrm{d} 0 \quad \text { ! left squark mass, 2nd generation } \\
& \operatorname{sql}(3)=400 . \mathrm{d} 0 \quad \text { ! left squark mass, 3rd generation } \\
& \operatorname{sqd}(1)=550 . \mathrm{d} 0 \quad \text { ! right down squark mass } \\
& \operatorname{sqd}(2)=550 . \mathrm{d} 0 \quad \text { ! right strange squark mass } \\
& \operatorname{sqd}(3)=300 \cdot \mathrm{d} 0 \quad ! \text { right sbottom mass } \\
& \mathrm{squ}(1)=450 . \mathrm{d} 0 \quad \text { ! right up squark mass } \\
& \mathrm{squ}(2)=450 \cdot \mathrm{d} 0 \quad \text { ! right charm squark mass } \\
& \mathrm{squ}(3)=200 \cdot \mathrm{d} 0 \quad \text { ! right stop mass } \\
& \operatorname{asd}(2)=(1 . \mathrm{d} 0,0 . \mathrm{d} 0) \quad ! \text { down squark } L R \text { mixing, 2nd generation } \\
& \operatorname{asd}(3)=(1 . \mathrm{d} 0,0 . \mathrm{d} 0) \quad ! \text { down squark } L R \text { mixing, 3rd generation } \\
& \operatorname{asu}(1)=(1 . \mathrm{d} 0,0 . \mathrm{d} 0) \quad ! \text { up squark } L R \text { mixing, } 1 \text { st generation } \\
& \operatorname{asu}(2)=(1 . \mathrm{d} 0,0 . \mathrm{d} 0) \quad ! \text { up squark } L R \text { mixing, 2nd generation } \\
& \operatorname{asu}(3)=(1 . \mathrm{d} 0,0 . \mathrm{d} 0) \quad ! \text { up squark LR mixing, 3rd generation }
\end{aligned}
$$

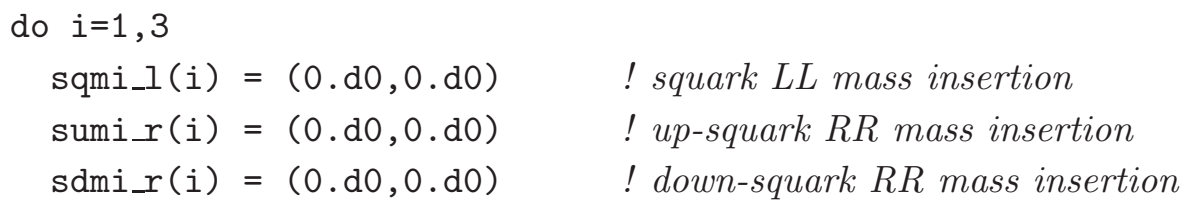


sqmi_l $(2)=(2 . \mathrm{d}-2,-1 . \mathrm{d}-2) \quad$ ! example, non-vanishing LL 23 entry

Squark LR mass insertions, non-hermitian in general

do $i=1,3$

do $j=1,3$

sumi_lr $(i, j)=(0 . \mathrm{d} 0,0 . \mathrm{d} 0)$ ! up-squark LR ij mass insertion

sdmi_lr $(i, j)=(0 . \mathrm{d} 0,0 . \mathrm{d} 0) !$ down-squark LR ij mass insertion

end do

end do

$c$

Calculate squark physical masses and mixing angles

call init_squark_sector (sql, squ, sqd, asu, asd, ierr, sqmi_, sumi_r,

\$ sdmi_r,sumi_lr,sdmi_lr)

if (ierr.ne.0) stop 'negative tree level squark mass ${ }^{2}$ ?'

c reset status of physical Higgs mass after parameter changes

call reset_phys_data

c Neutral CP-even Higgs masses in the 1-loop Effective Potential Approximation.

$c \quad$ Only real mu, $A_{-} t, A_{-} b$ allowed - replace $x \rightarrow a b s(x)$

call fcorr EPA (tanbe, pm, top, abs (amue), sql (3), sqd (3), squ (3),

$\$ \quad \operatorname{abs}(\operatorname{asd}(3)), \operatorname{abs}(\operatorname{asu}(3))$, ierr $)$

if (ierr.ne.0) stop 'negative 1-loop EPA CP-even Higgs mass²?'

100 continue !!! End of input section !!!

c Control output: Lagrangian parameters and tree level masses written on file mssm_data.txt

if $1=1 \quad$ ! output file number

open (ifl,file='mssm_data.txt', status='unknown')

call print_MSSM_par(ifl) ! Lagrangian parameters

call print_MSSM_masses(ifl) ! tree level physical masses

close(ifl)

c Results for implemented rare decays:

write $(*, 99)$ 'Electric dipole moments:'

write $(*, 99)$ 'Electron EDM = ', edm_l(1)

write $(*, 99)$ 'Muon EDM $=$ ', edm_l (2)

write $(*, 99)$ ' Tau EDM $=$ ', edm_l(3)

write $(*, 99)$ 'Neutron $\operatorname{EDM}=$ ', edm_n()

write $(*, 99)$ 'Neutrino $\mathrm{K}$ decays:'

call k_pivv(br_k0,br_kp)

write $(*, 99)^{\prime} \mathrm{BR}\left(\mathrm{K}_{L}^{0} \rightarrow \mathrm{pi}^{0} \mathrm{vv}\right)=$,, $\mathrm{br} \_\mathrm{k} 0$

write $(*, 99)^{\prime} \mathrm{BR}\left(\mathrm{K}^{+} \rightarrow \mathrm{pi}^{+} \mathrm{vv}\right)=$ ', $\mathrm{br \_ kp}$

write $(*, 99)$ 'Leptonic B decays:'

write $(*, 99)$ 'BR(B_d $\left.\rightarrow \mathrm{mu}^{+} \mathrm{mu}^{-}\right)=$,, $\mathrm{b} \_l l(3,1,2,2)$

write $(*, 99)$ 'BR(B_s $\left.\rightarrow \mathrm{mu}^{+} \mathrm{mu}^{-}\right)=$', b_ll $(3,2,2,2)$ 


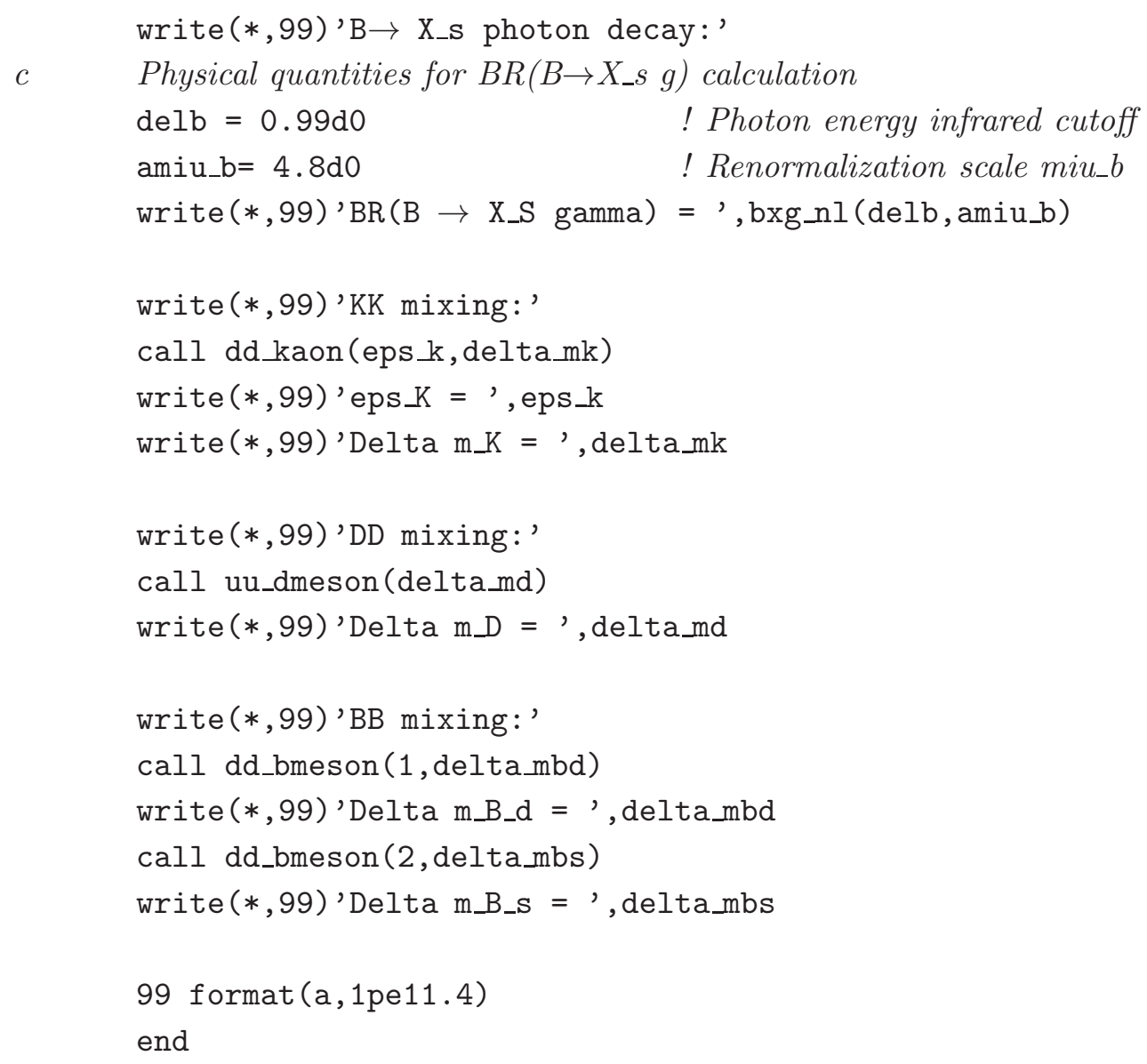

\section{Example of SUSY_FLAVOR input file}

By default, the driver file susy_flavor. f reads input parameters from the file susy_flavor.in. Below we provide an example input file defining a set of parameters equivalent to those in the driver file presented in Appendix B.

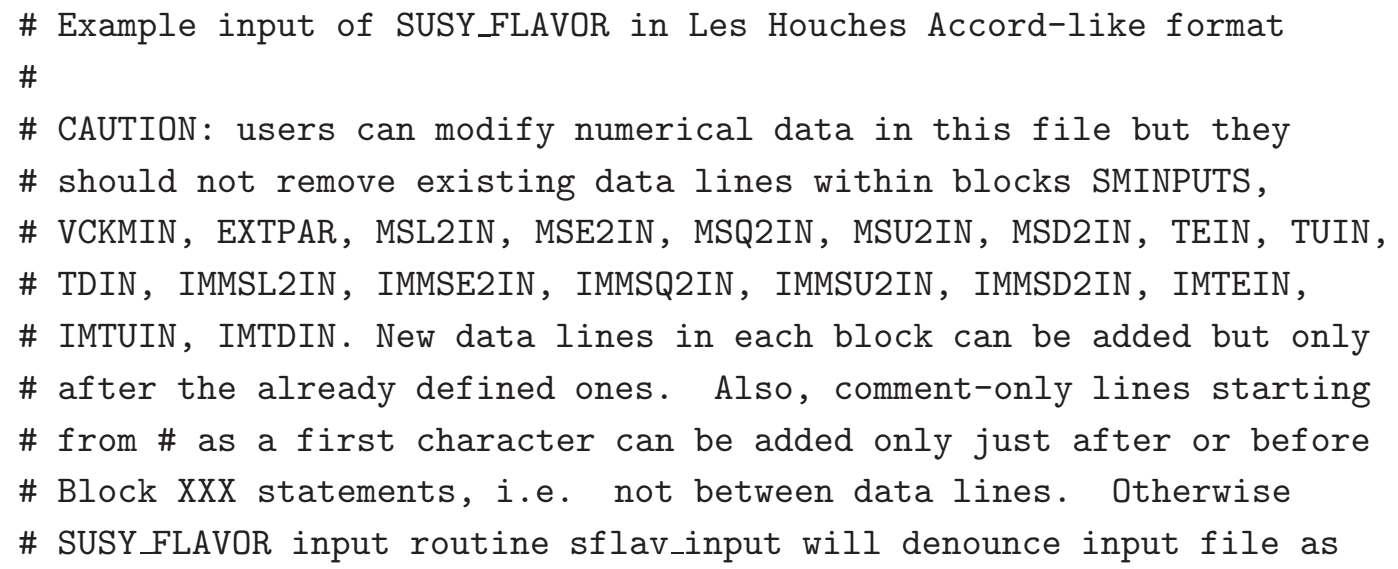




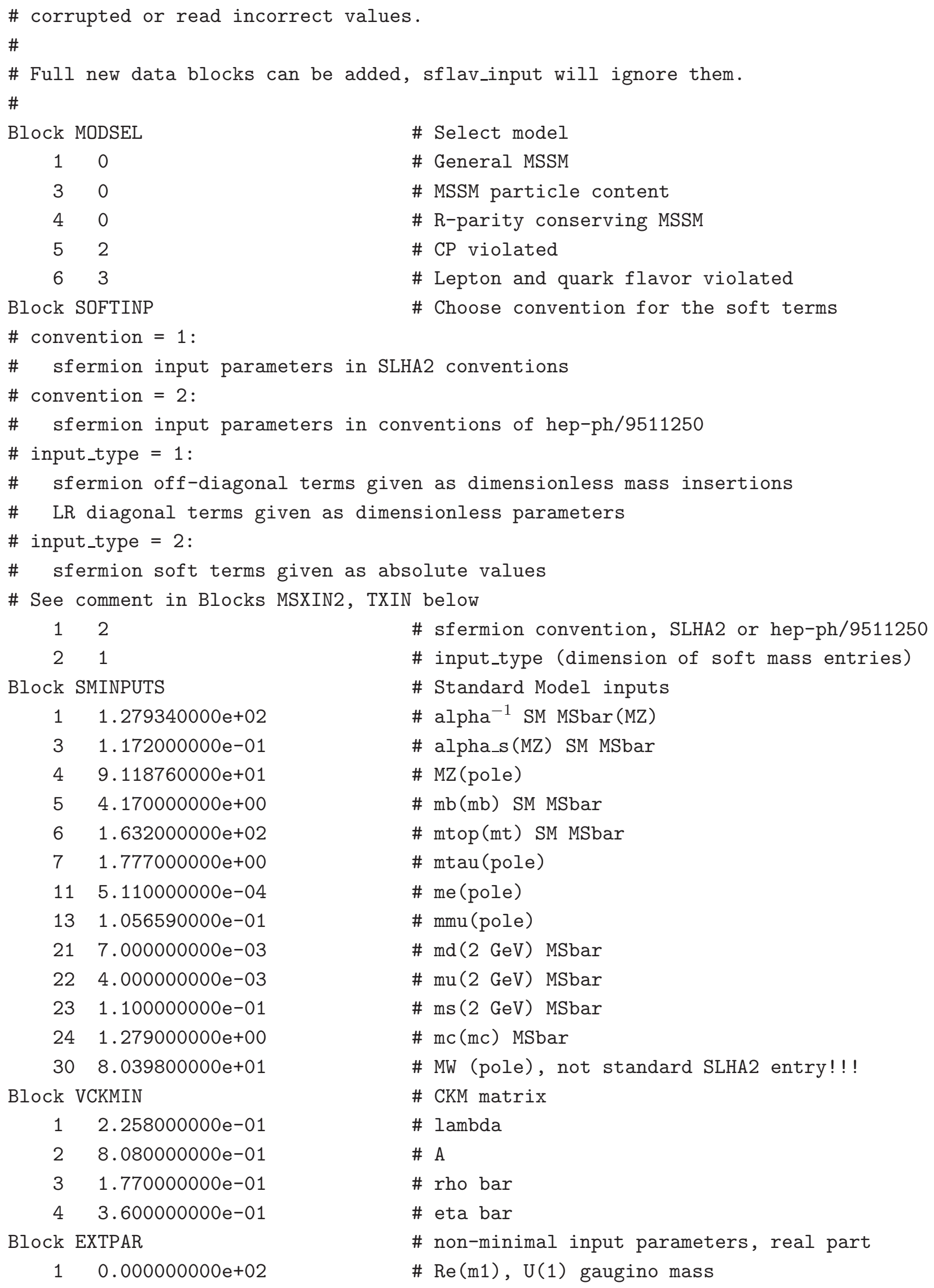




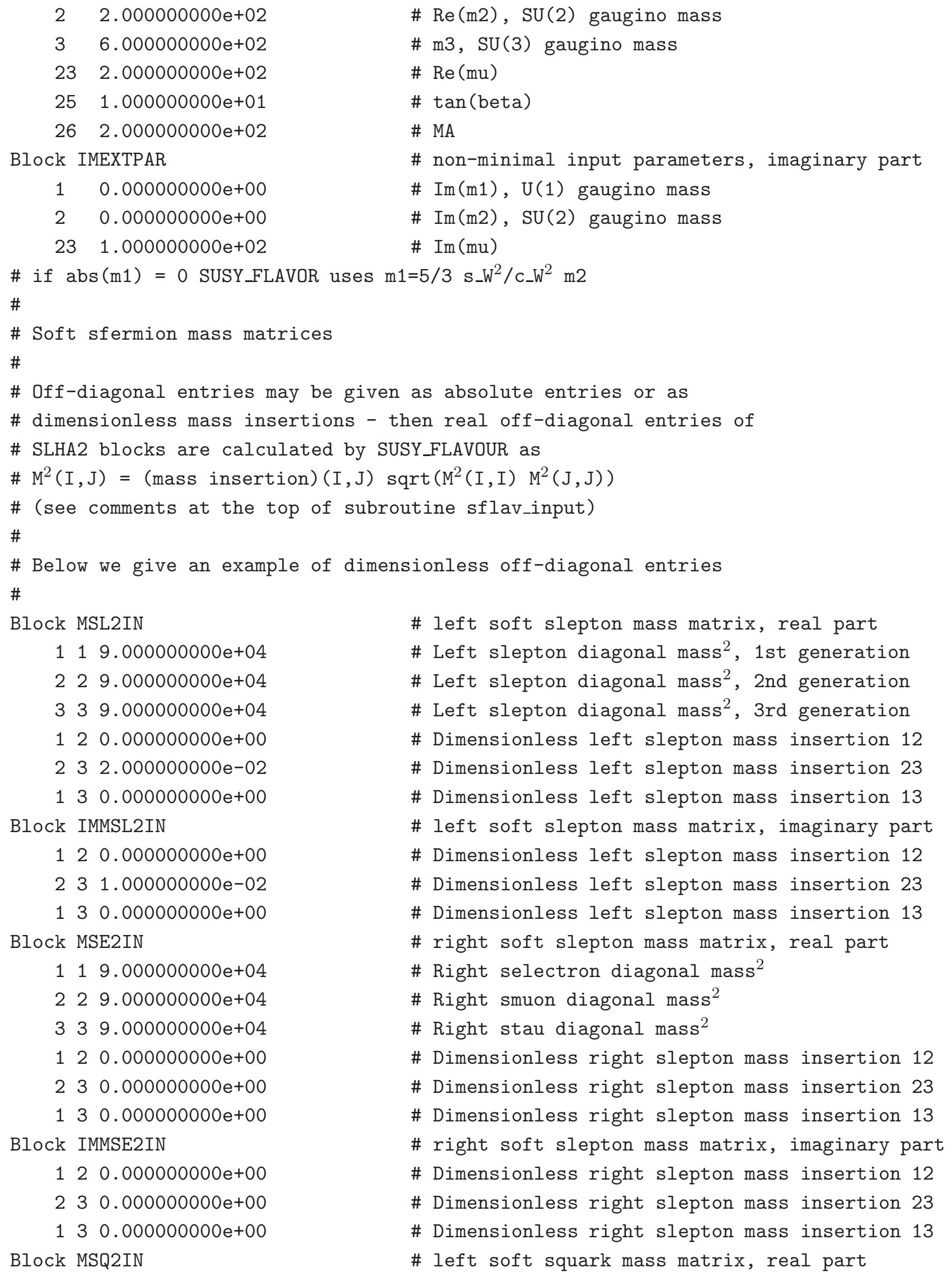

$119.000000000 \mathrm{e}+04$

$229.000000000 e+04$

$339.000000000 \mathrm{e}+04$

$120.000000000 \mathrm{e}+00$

$232.000000000 e-02$

$130.000000000 \mathrm{e}+00$

Block IMMSL2IN

$120.000000000 \mathrm{e}+00$

$231.000000000 \mathrm{e}-02$

$130.000000000 \mathrm{e}+00$

Block MSE2IN

$119.000000000 \mathrm{e}+04$

$229.000000000 \mathrm{e}+04$

$339.000000000 \mathrm{e}+04$

$120.000000000 \mathrm{e}+00$

$230.000000000 \mathrm{e}+00$

$130.000000000 \mathrm{e}+00$

Block IMMSE2IN

$120.000000000 \mathrm{e}+00$

$230.000000000 \mathrm{e}+00$

$130.000000000 \mathrm{e}+00$

Block MSQ2IN

\# left soft slepton mass matrix, real part

\# Left slepton diagonal mass ${ }^{2}$, 1st generation \# Left slepton diagonal mass ${ }^{2}$, 2nd generation \# Left slepton diagonal mass ${ }^{2}$, 3rd generation \# Dimensionless left slepton mass insertion 12 \# Dimensionless left slepton mass insertion 23 \# Dimensionless left slepton mass insertion 13 \# left soft slepton mass matrix, imaginary part \# Dimensionless left slepton mass insertion 12 \# Dimensionless left slepton mass insertion 23 \# Dimensionless left slepton mass insertion 13 \# right soft slepton mass matrix, real part \# Right selectron diagonal mass ${ }^{2}$

\# Right smuon diagonal mass ${ }^{2}$

\# Right stau diagonal mass ${ }^{2}$

\# Dimensionless right slepton mass insertion 12

\# Dimensionless right slepton mass insertion 23

\# Dimensionless right slepton mass insertion 13 \# right soft slepton mass matrix, imaginary part \# Dimensionless right slepton mass insertion 12 \# Dimensionless right slepton mass insertion 23 \# Dimensionless right slepton mass insertion 13 \# left soft squark mass matrix, real part 
$112.500000000 \mathrm{e}+05$

$222.500000000 \mathrm{e}+05$

$331.600000000 \mathrm{e}+05$

$120.000000000 \mathrm{e}+00$

$232.000000000 e-02$

$130.000000000 \mathrm{e}+00$

Block IMMSQ2IN

$120.000000000 \mathrm{e}+00$

$23-1.000000000 e-02$

$130.000000000 \mathrm{e}+00$

Block MSU2IN

$112.025000000 \mathrm{e}+05$

$222.025000000 \mathrm{e}+05$

$334.000000000 \mathrm{e}+04$

$120.000000000 \mathrm{e}+00$

$230.000000000 \mathrm{e}+00$

$130.000000000 \mathrm{e}+00$

Block IMMSU2IN

$120.000000000 \mathrm{e}+00$

$230.000000000 \mathrm{e}+00$

$130.000000000 \mathrm{e}+00$
\# Left squark diagonal mass ${ }^{2}$, 1st generation \# Left squark diagonal mass ${ }^{2}$, 2nd generation \# Left squark diagonal mass ${ }^{2}$, 3rd generation \# Dimensionless left squark mass insertion 12 \# Dimensionless left squark mass insertion 23 \# Dimensionless left squark mass insertion 13 \# left soft squark mass matrix, imaginary part \# Dimensionless left squark mass insertion 12 \# Dimensionless left squark mass insertion 23 \# Dimensionless left squark mass insertion 13 \# right soft up-squark mass matrix, real part \# Right u-squark diagonal mass $^{2}$

\# Right c-squark diagonal mass ${ }^{2}$

\# Right stop diagonal mass ${ }^{2}$

\# Dimensionless right up-squark mass insertion 12

\# Dimensionless right up-squark mass insertion 23

\# Dimensionless right up-squark mass insertion 13

\# right soft up-squark mass matrix, imaginary part

\# Dimensionless right up-squark mass insertion 12

\# Dimensionless right up-squark mass insertion 23

\# Dimensionless right up-squark mass insertion 13

Block MSD2IN \# right soft down-squark mass matrix, real part

$113.025000000 \mathrm{e}+05$

$223.025000000 \mathrm{e}+05$

$339.000000000 \mathrm{e}+04$

$120.000000000 \mathrm{e}+00$

$230.000000000 \mathrm{e}+00$

$130.000000000 \mathrm{e}+00$

Block IMMSD2IN

$120.000000000 \mathrm{e}+00$

$230.000000000 e+00$

$130.000000000 \mathrm{e}+00$
\# Right d-squark diagonal mass ${ }^{2}$

\# Right s-squark diagonal mass ${ }^{2}$

\# Right sbottom diagonal mass ${ }^{2}$

\# Dimensionless right down-squark mass insertion 12

\# Dimensionless right down-squark mass insertion 23

\# Dimensionless right down-squark mass insertion 13

\# right soft down-squark mass matrix, imaginary part

\# Dimensionless right down-squark mass insertion 12

\# Dimensionless right down-squark mass insertion 23

\# Dimensionless right down-squark mass insertion 13

\#

\# Soft sfermion trilinear mixing matrices

\#

\# LR mixing parameters can be given as absolute entries or as

\# dimensionless diagonal A-terms and dimensionless ff-diagonal mass

\# insertions - see comments at the top of subroutine sflav_input

$\#$

\# Below we give an example of dimensionless A terms.

\#

\# Diagonal entries below are dimensionless "A parameters"

\# Diagonal entries of SLHA2 LR blocks are calculated by SUSYFLAVOUR as

$\# \mathrm{TL}(I, I)=\mathrm{AL}(I, I)$ Yukawa $L(I) \operatorname{sqrt}\left(\mathrm{ML}^{2}(I, I) * \mathrm{ME}^{2}(I, I)\right)$ 


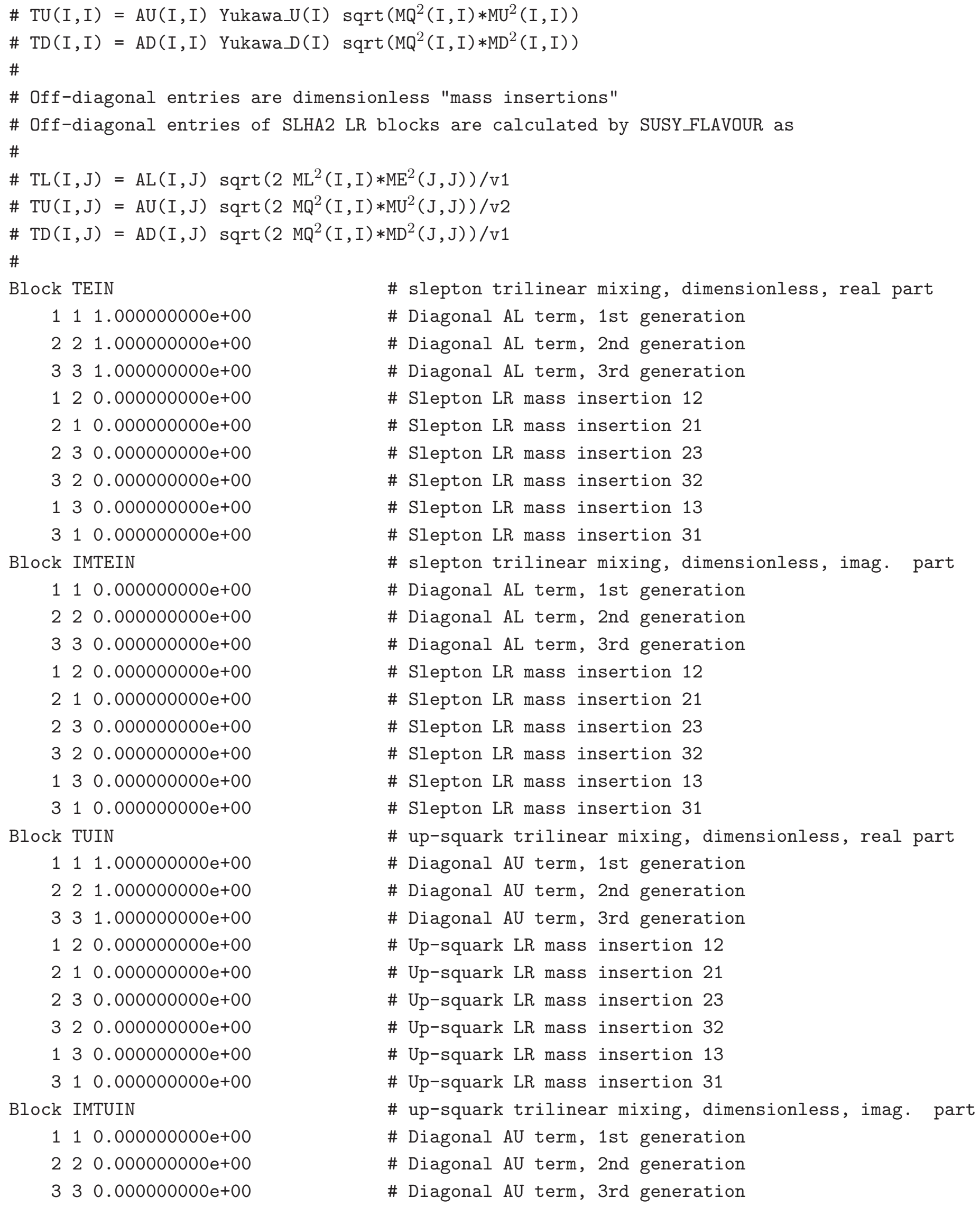

$111.000000000 \mathrm{e}+00$

$221.000000000 \mathrm{e}+00$

$331.000000000 \mathrm{e}+00$

$120.000000000 \mathrm{e}+00$

$210.000000000 \mathrm{e}+00$

$230.000000000 \mathrm{e}+00$

$320.000000000 \mathrm{e}+00$

$130.000000000 \mathrm{e}+00$

$310.000000000 \mathrm{e}+00$

Block IMTUIN

$110.000000000 \mathrm{e}+00$

$220.000000000 \mathrm{e}+00$

$330.000000000 \mathrm{e}+00$

\# slepton trilinear mixing, dimensionless, real part

\# Diagonal AL term, 1st generation

\# Diagonal AL term, 2nd generation

\# Diagonal AL term, 3rd generation

\# Slepton LR mass insertion 12

\# Slepton LR mass insertion 21

\# Slepton LR mass insertion 23

\# Slepton LR mass insertion 32

\# Slepton LR mass insertion 13

\# Slepton LR mass insertion 31

\# slepton trilinear mixing, dimensionless, imag. part

\# Diagonal AL term, 1st generation

\# Diagonal AL term, 2nd generation

\# Diagonal AL term, 3rd generation

\# Slepton LR mass insertion 12

\# Slepton LR mass insertion 21

\# Slepton LR mass insertion 23

\# Slepton LR mass insertion 32

\# Slepton LR mass insertion 13

\# Slepton LR mass insertion 31

\# up-squark trilinear mixing, dimensionless, real part

\# Diagonal AU term, 1st generation

\# Diagonal AU term, 2nd generation

\# Diagonal AU term, 3rd generation

\# Up-squark LR mass insertion 12

\# Up-squark LR mass insertion 21

\# Up-squark LR mass insertion 23

\# Up-squark LR mass insertion 32

\# Up-squark LR mass insertion 13

\# Up-squark LR mass insertion 31

\# up-squark trilinear mixing, dimensionless, imag. part

\# Diagonal AU term, 1st generation

\# Diagonal AU term, 2nd generation

\# Diagonal AU term, 3rd generation 


$\begin{array}{rrr}1 & 2 & 0.000000000 \mathrm{e}+00 \\ 2 & 1 & 0.000000000 \mathrm{e}+00 \\ 2 & 3 & 0.000000000 \mathrm{e}+00 \\ 3 & 2 & 0.000000000 \mathrm{e}+00 \\ 1 & 3 & 0.000000000 \mathrm{e}+00 \\ 3 & 1 & 0.000000000 \mathrm{e}+00 \\ \text { Block } & \text { TDIN } \\ 1 & 1 & 1.000000000 \mathrm{e}+00 \\ 2 & 2 & 1.000000000 \mathrm{e}+00 \\ 3 & 3 & 1.000000000 \mathrm{e}+00 \\ 1 & 2 & 0.000000000 \mathrm{e}+00 \\ 2 & 1 & 0.000000000 \mathrm{e}+00 \\ 2 & 3 & 0.000000000 \mathrm{e}+00 \\ 3 & 2 & 0.000000000 \mathrm{e}+00 \\ 1 & 3 & 0.000000000 \mathrm{e}+00 \\ 3 & 1 & 0.000000000 \mathrm{e}+00 \\ \text { Block } & \text { IMTDIN } \\ 1 & 1 & 0.000000000 \mathrm{e}+00 \\ 2 & 2 & 0.000000000 \mathrm{e}+00 \\ 3 & 3 & 0.000000000 \mathrm{e}+00 \\ 1 & 2 & 0.000000000 \mathrm{e}+00 \\ 2 & 1 & 0.000000000 \mathrm{e}+00 \\ 2 & 3 & 0.000000000 \mathrm{e}+00 \\ 3 & 2 & 0.000000000 \mathrm{e}+00 \\ 1 & 3 & 0.000000000 \mathrm{e}+00 \\ 3 & 1 & 0.000000000 \mathrm{e}+00\end{array}$

\# Up-squark LR mass insertion 12

\# Up-squark LR mass insertion 21

\# Up-squark LR mass insertion 23

\# Up-squark LR mass insertion 32

\# Up-squark LR mass insertion 13

\# Up-squark LR mass insertion 31

\# down-squark trilinear mixing, dimensionless, real part

\# Diagonal AD term, 1st generation

\# Diagonal AD term, 2nd generation

\# Diagonal AD term, 3rd generation

\# Down-squark LR mass insertion 12

\# Down-squark LR mass insertion 21

\# Down-squark LR mass insertion 23

\# Down-squark LR mass insertion 32

\# Down-squark LR mass insertion 13

\# Down-squark LR mass insertion 31

\# down-squark trilinear mixing, dimensionless, imag. part

\# Diagonal AD term, 1st generation

\# Diagonal AD term, 2nd generation

\# Diagonal AD term, 3rd generation

\# Down-squark LR mass insertion 12

\# Down-squark LR mass insertion 21

\# Down-squark LR mass insertion 23

\# Down-squark LR mass insertion 32

\# Down-squark LR mass insertion 13

\# Down-squark LR mass insertion 31

\section{Example of SUSY_FLAVOR output}

The parameters defined inside the driver program in Appendix $\mathrm{B}$ and in the input file listed in Appendix $[$ should produce identical output. We enclose it here so that SUSY_FLAVOR users can check that the program gives the same result on their own computers and FORTRAN compilers.

The driver file susy_flavor.f writes the MSSM Lagrangian parameters and tree-level particle masses to the file mssm_data.txt. For the parameters defined in Appendices B and $\mathrm{C}$ one has:

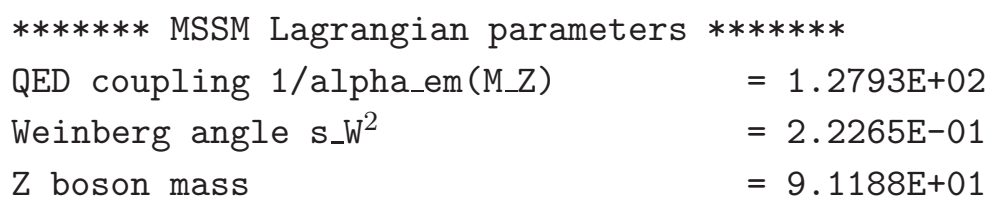




\begin{tabular}{|c|c|c|c|c|}
\hline \multicolumn{3}{|l|}{ W boson mass } & $=8.0398 \mathrm{E}+01$ & \\
\hline QCD coupling & alpha_s (M_Z) & & $=1 \cdot 1720 \mathrm{E}-01$ & \\
\hline \multicolumn{3}{|c|}{ Higgs mixing parameter mu (complex) } & $=2.0000 \mathrm{E}+02$ & $1.0000 \mathrm{E}+02$ \\
\hline \multicolumn{3}{|c|}{ Higgs soft mixing parameter $\mathrm{m}_{12}^{2}$} & $=-3.9604 \mathrm{E}+03$ & \\
\hline \multicolumn{3}{|c|}{ Higgs soft masses $\mathrm{m}_{H_{1}}^{2}, \mathrm{~m}_{H_{2}}^{2}$} & $=-6.3208 \mathrm{E}+03$ & $-5.3679 E+04$ \\
\hline \multicolumn{3}{|c|}{ U(1) gaugino mass (complex) } & $=9.5472 \mathrm{E}+01$ & $0.0000 \mathrm{E}+00$ \\
\hline \multicolumn{3}{|c|}{ SU(2) gaugino mass (complex) } & $=2.0000 \mathrm{E}+02$ & $0.0000 \mathrm{E}+00$ \\
\hline \multicolumn{3}{|c|}{$\mathrm{SU}$ (3) gaugino mass (real) } & $=6.0000 \mathrm{E}+02$ & \\
\hline \multicolumn{5}{|c|}{ Left slepton mass matrix, real part: } \\
\hline \multicolumn{5}{|c|}{$9.00009 \mathrm{E}+04 \quad 0.00000 \mathrm{E}+00 \quad 0.00000 \mathrm{E}+00$} \\
\hline $0.00000 \mathrm{E}+00$ & $9.00018 \mathrm{E}+04$ & 1.8 & $004 \mathrm{E}+03$ & \\
\hline $0.00000 \mathrm{E}+00$ & $1.80004 \mathrm{E}+03$ & 9.0 & $027 \mathrm{E}+04$ & \\
\hline \multicolumn{5}{|c|}{ Left slepton mass matrix, imaginary part: } \\
\hline $0.00000 \mathrm{E}+00$ & $0.00000 \mathrm{E}+00$ & 0.0 & $000 \mathrm{E}+00$ & \\
\hline$-0.00000 E+00$ & $0.00000 \mathrm{E}+00$ & 9.0 & $022 \mathrm{E}+02$ & \\
\hline$-0.00000 E+00$ & $-9.00022 \mathrm{E}+02$ & 0.0 & $000 E+00$ & \\
\hline \multicolumn{5}{|c|}{ Right slepton mass matrix, real part: } \\
\hline \multicolumn{2}{|l|}{$8.99991 \mathrm{E}+04$} & 0.0 & $000 \mathrm{E}+00$ & \\
\hline $0.00000 \mathrm{E}+00$ & $8.99982 E+04$ & 0.0 & $000 \mathrm{E}+00$ & \\
\hline $0.00000 \mathrm{E}+00$ & $0.00000 \mathrm{E}+00$ & 8.9 & $973 E+04$ & \\
\hline \multicolumn{5}{|c|}{ Right slepton mass matrix, imaginary part: } \\
\hline $0.00000 \mathrm{E}+00$ & $0.00000 \mathrm{E}+00$ & 0.0 & $000 \mathrm{E}+00$ & \\
\hline $0.00000 \mathrm{E}+00$ & $0.00000 \mathrm{E}+00$ & 0.0 & $000 \mathrm{E}+00$ & \\
\hline $0.00000 \mathrm{E}+00$ & $0.00000 \mathrm{E}+00$ & 0.0 & $000 \mathrm{E}+00$ & \\
\hline \multicolumn{5}{|c|}{ Slepton LR mixing matrix, real part: } \\
\hline$-9.00010 E-03$ & $0.00000 \mathrm{E}+00$ & 0.0 & $000 \mathrm{E}+00$ & \\
\hline $0.00000 \mathrm{E}+00$ & $-1.86094 \mathrm{E}+00$ & 0.0 & $000 \mathrm{E}+00$ & \\
\hline $0.00000 \mathrm{E}+00$ & $0.00000 \mathrm{E}+00$ & -3 & $2978 \mathrm{E}+01$ & \\
\hline Slepton LR mix & xing matrix, imag: & nary & part: & \\
\hline $0.00000 \mathrm{E}+00$ & $0.00000 \mathrm{E}+00$ & 0.0 & $000 E+00$ & \\
\hline $0.00000 \mathrm{E}+00$ & $0.00000 \mathrm{E}+00$ & 0.0 & $000 E+00$ & \\
\hline $0.00000 \mathrm{E}+00$ & $0.00000 \mathrm{E}+00$ & 0.0 & $000 E+00$ & \\
\hline Left squark ma & ass matrix, real & art: & & \\
\hline $2.50003 E+05$ & $0.00000 \mathrm{E}+00$ & 0.0 & $000 \mathrm{E}+00$ & \\
\hline $0.00000 \mathrm{E}+00$ & $2.50005 \mathrm{E}+05$ & 4.0 & $010 \mathrm{E}+03$ & \\
\hline $0.00000 \mathrm{E}+00$ & $4.00010 \mathrm{E}+03$ & 1.6 & $005 E+05$ & \\
\hline Left squark ma & ss matrix, imagir & $\operatorname{ary}$ & art: & \\
\hline $0.00000 \mathrm{E}+00$ & $0.00000 \mathrm{E}+00$ & 0.0 & $000 E+00$ & \\
\hline
\end{tabular}



$0.00000 \mathrm{E}+00$
$0.00000 \mathrm{E}+00$
$-2.00005 E+03$
$0.00000 \mathrm{E}+00$
$2.00005 \mathrm{E}+03$
$0.00000 \mathrm{E}+00$

Right up-squark mass matrix, real part:
$2.02498 \mathrm{E}+05$
$0.00000 \mathrm{E}+00$
$0.00000 \mathrm{E}+00$
$0.00000 \mathrm{E}+00$
$2.02496 \mathrm{E}+05$
$0.00000 \mathrm{E}+00$
$0.00000 \mathrm{E}+00$
$0.00000 \mathrm{E}+00$
$3.99988 \mathrm{E}+04$

Right up-squark mass matrix, imaginary part:
$0.00000 \mathrm{E}+00$
$0.00000 \mathrm{E}+00$
$0.00000 \mathrm{E}+00$
$0.00000 \mathrm{E}+00$
$0.00000 \mathrm{E}+00$
$0.00000 \mathrm{E}+00$
$0.00000 \mathrm{E}+00$
$0.00000 \mathrm{E}+00$
$0.00000 \mathrm{E}+00$

Right down-squark mass matrix, real part:
3. 02497E+05
$0.00000 \mathrm{E}+00$
$0.00000 \mathrm{E}+00$
$0.00000 \mathrm{E}+00$
3. 02494E+05
$0.00000 \mathrm{E}+00$
$0.00000 \mathrm{E}+00$
$0.00000 \mathrm{E}+00$
8. 99973E+04

Right down-squark mass matrix, imaginary part:
$0.00000 \mathrm{E}+00$
$0.00000 \mathrm{E}+00$
$0.00000 \mathrm{E}+00$
$0.00000 \mathrm{E}+00$
$0.00000 \mathrm{E}+00$
$0.00000 \mathrm{E}+00$
$0.00000 \mathrm{E}+00$
$0.00000 \mathrm{E}+00$
$0.00000 \mathrm{E}+00$

Up-squark LR mixing matrix, real part:
$6.18168 \mathrm{E}-03$
$0.00000 \mathrm{E}+00$
$0.00000 \mathrm{E}+00$
$0.00000 \mathrm{E}+00$
$1.79332 \mathrm{E}+00$
$0.00000 \mathrm{E}+00$
$0.00000 \mathrm{E}+00$
$0.00000 \mathrm{E}+00$
$2.71002 \mathrm{E}+02$
Up-squark LR mixing matrix, imaginary part:

$\begin{array}{lll}0.00000 \mathrm{E}+00 & 0.00000 \mathrm{E}+00 & 0.00000 \mathrm{E}+00 \\ 0.00000 \mathrm{E}+00 & 0.00000 \mathrm{E}+00 & 0.00000 \mathrm{E}+00 \\ 0.00000 \mathrm{E}+00 & 0.00000 \mathrm{E}+00 & 0.00000 \mathrm{E}+00\end{array}$

Down-squark LR mixing matrix, real part:

$\begin{array}{lll}-1.19597 \mathrm{E}-01 & 0.00000 \mathrm{E}+00 & 0.00000 \mathrm{E}+00 \\ 0.00000 \mathrm{E}+00 & -1.87938 \mathrm{E}+00 & 0.00000 \mathrm{E}+00 \\ 0.00000 \mathrm{E}+00 & 0.00000 \mathrm{E}+00 & -5.56628 \mathrm{E}+01\end{array}$

Down-squark LR mixing matrix, imaginary part:
$0.00000 \mathrm{E}+00$
$0.00000 \mathrm{E}+00$
$0.00000 \mathrm{E}+00$
$0.00000 \mathrm{E}+00$
$0.00000 \mathrm{E}+00$
$0.00000 \mathrm{E}+00$
$0.00000 \mathrm{E}+00$
$0.00000 \mathrm{E}+00$
$0.00000 \mathrm{E}+00$

****** Particle masses in $\mathrm{GeV}$ :

* Fermion masses *

Charged lepton masses

Running u quark masses at $m_{t}$ scale

Running d quark masses at $m_{t}$ scale
5.110E-04 1.057E-01 1.777E+00
$2.220 \mathrm{E}-03 \quad 6.440 \mathrm{E}-01 \quad 1.632 \mathrm{E}+02$
$3.885 \mathrm{E}-03 \quad 6.104 \mathrm{E}-02 \quad 2.737 \mathrm{E}+00$ 


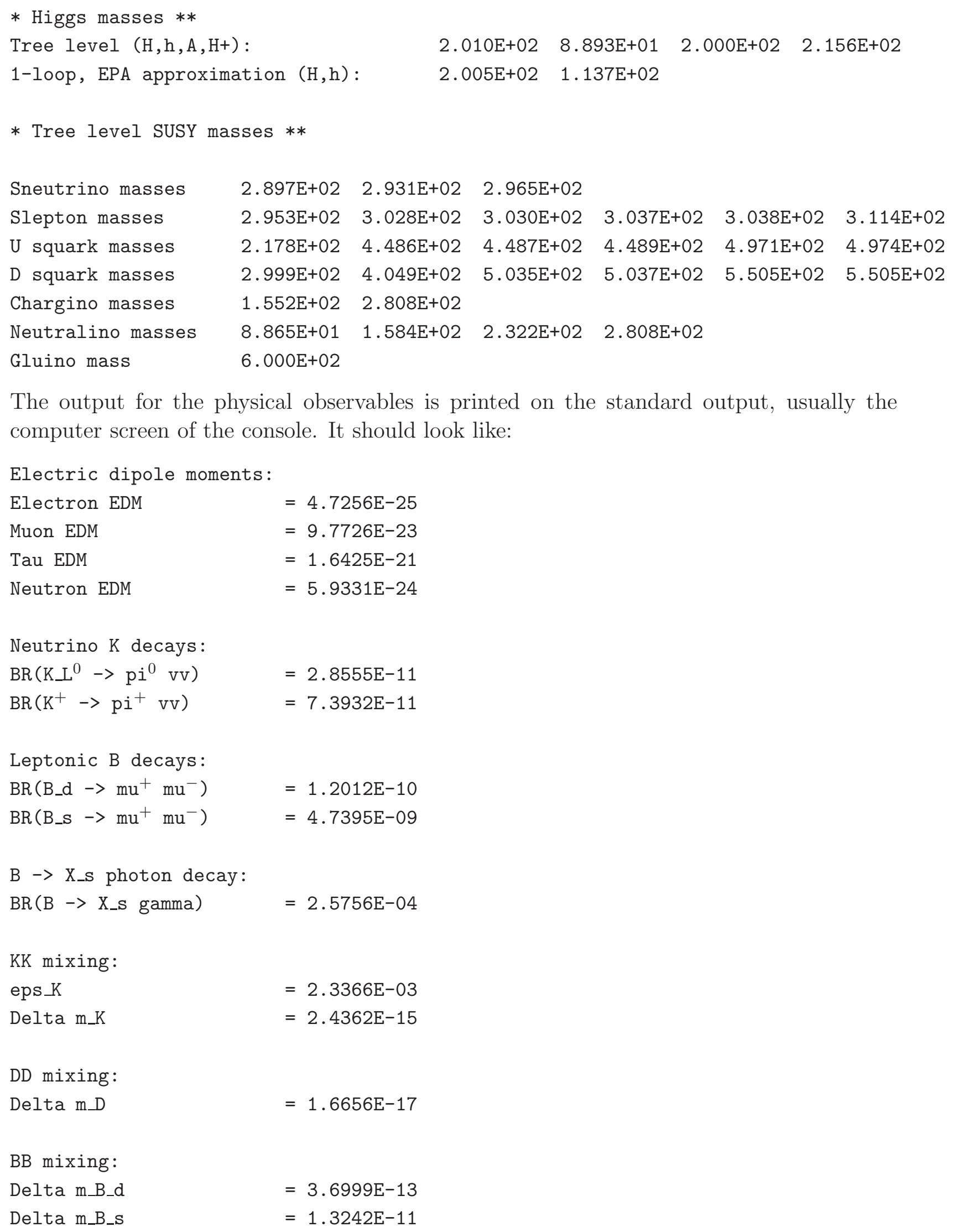

The output for the physical observables is printed on the standard output, usually the computer screen of the console. It should look like:

Electric dipole moments: 


\section{References}

[1] For reviews, see for example,

H. P. Nilles, Phys. Rept. 110, 1 (1984).

H. E. Haber and G. L. Kane, Phys. Rept. 117, 75 (1985).

S. P. Martin, arXiv:hep-ph/9709356.

S. Weinberg, "The quantum theory of fields. Vol. 3: Supersymmetry," Cambridge, UK: Univ. Pr. (2000) $419 p$

[2] For a recent review, A. J. Buras, "Flavour Theory: 2009," arXiv:0910.1032 [hep-ph].

M. Artuso et al., Eur. Phys. J. C 57 (2008) 309 [arXiv:0801.1833 [hep-ph]].

[3] M. Misiak, S. Pokorski and J. Rosiek, "Supersymmetry and the FCNC effects" Adv. Ser. Direct. High Energy Phys. 15 (1998) 795 arXiv:hep-ph/9703442.

[4] S. Pokorski, J. Rosiek and C. A. Savoy, Nucl. Phys. B 570 (2000) 81 arXiv:hep-ph/9906206.

[5] J. Rosiek, Acta Phys. Polon. B 30 (1999) 3379.

[6] A. J. Buras, P. H. Chankowski, J. Rosiek and L. Slawianowska, Nucl. Phys. B 619 (2001) 434 arXiv:hep-ph/0107048.

[7] J. Rosiek, arXiv:hep-ph/0108226.

[8] A. J. Buras, P. H. Chankowski, J. Rosiek and L. Slawianowska, Phys. Lett. B 546 (2002) 96 arXiv:hep-ph/0207241.

[9] P. H. Chankowski and J. Rosiek, Acta Phys. Polon. B 33 (2002) 2329 arXiv:hep-ph/0207242.

[10] A. J. Buras, P. H. Chankowski, J. Rosiek and L. Slawianowska, Nucl. Phys. B 659 (2003) 3 arXiv:hep-ph/0210145.

[11] A. J. Buras, T. Ewerth, S. Jager and J. Rosiek, Nucl. Phys. B 714 (2005) 103 arXiv:hep-ph/0408142.

[12] A. Dedes, J. Rosiek and P. Tanedo, Phys. Rev. D 79 (2009) 055006 arXiv:0812.4320 [hep-ph]].

[13] J. Rosiek, arXiv:0911.3339 [hep-ph].

[14] J. Rosiek, Phys. Rev. D 41 (1990) 3464; erratum arXiv:hep-ph/9511250.

[15] A. Dedes, J. R. Ellis and M. Raidal, Phys. Lett. B 549 (2002) 159 arXiv:hep-ph/0209207. 
[16] B. C. Regan, E. D. Commins, C. J. Schmidt and D. DeMille, Phys. Rev. Lett. 88, 071805 (2002).

[17] R. McNabb [Muon g-2 Collaboration], arXiv:hep-ex/0407008.

[18] C. Amsler et al. [Particle Data Group], Phys. Lett. B 667 (2008) 1.

[19] C. A. Baker et al., Phys. Rev. Lett. 97, 131801 (2006) arXiv:hep-ex/0602020].

[20] J. K. Ahn et al. [E391a Collaboration], Phys. Rev. Lett. 100, 201802 (2008) arXiv:0712.4164 [hep-ex]].

[21] A. V. Artamonov et al. [E949 Collaboration], Phys. Rev. Lett. 101, 191802 (2008) arXiv:0808.2459 [hep-ex]].

[22] B. Aubert et al. [BaBar Collaboration], Phys. Rev. D 77 (2008) 032007 arXiv:0712.1516 [hep-ex]].

[23] T. Aaltonen et al. [CDF Collaboration], Phys. Rev. Lett. 100, 101802 (2008) arXiv:0712.1708 [hep-ex]]. Somewhat more relaxed bounds have been obtained by V. M. Abazov et al. [D0 Collaboration], Phys. Rev. D 76, 092001 (2007) arXiv:0707.3997 [hep-ex]].

[24] B. Aubert et al. [BABAR Collaboration], Phys. Rev. Lett. 96 (2006) 241802 arXiv:hep-ex/0511015.

[25] M. Acciarri et al. [L3 Collaboration], Phys. Lett. B 391 (1997) 474.

[26] E. Barberio et al. [Heavy Flavor Averaging Group], arXiv:0808.1297 [hep-ex].

[27] A. Abulencia et al. [CDF Collaboration], Phys. Rev. Lett. 97 (2006) 242003 arXiv:hep-ex/0609040.

[28] J. S. Lee, M. Carena, J. Ellis, A. Pilaftsis and C. E. M. Wagner, Comput. Phys. Commun. 180 (2009) 312 arXiv:0712.2360 [hep-ph]].

[29] G. Degrassi, P. Gambino and P. Slavich, Comput. Phys. Commun. 179, 759 (2008) arXiv:0712.3265 [hep-ph]].

[30] F. Mahmoudi, Comput. Phys. Commun. 180, 1579 (2009) arXiv:0808.3144 [hep-ph]].

[31] S. Heinemeyer, W. Hollik and G. Weiglein, Comput. Phys. Commun. 124, 76 (2000) arXiv:hep-ph/9812320.

[32] B. C. Allanach, Comput. Phys. Commun. 143, 305 (2002) arXiv:hep-ph/0104145.

[33] A. Djouadi, J. L. Kneur and G. Moultaka, Comput. Phys. Commun. 176, 426 (2007) arXiv:hep-ph/0211331.

[34] W. Porod, Comput. Phys. Commun. 153, 275 (2003) arXiv:hep-ph/0301101. 
[35] G. Belanger, F. Boudjema, A. Pukhov and A. Semenov, Comput. Phys. Commun. 177, 894 (2007).

[36] P. Gondolo, J. Edsjo, P. Ullio, L. Bergstrom, M. Schelke and E. A. Baltz, JCAP 0407 (2004) 008 arXiv:astro-ph/0406204.

[37] U. Ellwanger and C. Hugonie, Comput. Phys. Commun. 175 (2006) 290 arXiv:hep-ph/0508022.

[38] L. J. Hall and L. Randall, Phys. Rev. Lett. 65 (1990) 2939.

[39] A. Dedes and A. Pilaftsis, Phys. Rev. D 67 (2003) 015012 arXiv:hep-ph/0209306.

[40] A. Crivellin and U. Nierste, arXiv:0908.4404 [hep-ph].

[41] J. Foster, K. I. Okumura and L. Roszkowski, JHEP 0508 (2005) 094.

[42] B. Allanach et al., Comput. Phys. Commun. 180 (2009) 8 [arXiv:0801.0045 [hep-ph]].

[43] A. Dedes, H. Haber and J. Rosiek, JHEP 0711 (2007) 059 [arXiv:0707.3718 [hep-ph]].

[44] P. Z. Skands et al., JHEP 0407 (2004) 036 arXiv:hep-ph/0311123.

[45] G. Buchalla and A. J. Buras, Nucl. Phys. B548 (1999) 309.

[46] W. Altmannshofer, A. J. Buras, S. Gori, P. Paradisi and D. M. Straub, Nucl. Phys. B 830 (2010) 17 arXiv:0909.1333 [hep-ph]].

[47] A. Manohar, H. Georgi, Nucl. Phys. B234 (1984) 189.

[48] R. Arnowitt, J. Lopez and D.V. Nanopoulos Phys. Rev. D42 (1990) 2423; R. Arnowitt, M. Duff and K. Stelle, Phys. Rev. D43 (1991) 3085.

[49] F. Mescia and C. Smith, Phys. Rev. D 76 (2007) 034017 [arXiv:0705.2025 [hep-ph]].

[50] L. Wolfenstein, Phys. Rev. Lett. 51 (1983) 1945.

[51] G. Buchalla and A. J. Buras, Nucl. Phys. B412 (1994) 106.

[52] A. J. Buras, M. Gorbahn, U. Haisch and U. Nierste, Phys. Rev. Lett. 95 (2005) 261805 arXiv:hep-ph/0508165; J. Brod and M. Gorbahn, Phys. Rev. D 78 (2008) 034006 [arXiv:0805.4119 [hep-ph]]; G. Isidori, F. Mescia and C. Smith, Nucl. Phys. B 718 (2005) 319 arXiv:hep-ph/0503107.

[53] A. J. Buras, S. Jager and J. Urban, Nucl. Phys. B 605 (2001) 600 arXiv:hep-ph/0102316.

[54] A. J. Buras, M. Jamin and P. H. Weisz, Nucl. Phys. B347 (1990) 491; J. Urban, F. Krauss, U. Jentschura and G. Soff, Nucl. Phys. B523 (1998) 40.

[55] S. Herrlich and U. Nierste, Nucl. Phys. B419 (1994) 292; S. Herrlich and U. Nierste, Phys. Rev. D52 (1995) 6505; Phys. Rev. D476 (1996) 27. 
[56] A. J. Buras, Les Houches Lectures "Probing the Standard Model of Particle Interactions", F. David and R. Gupta, eds, Elsevier Science B.V., arXiv:hep-ph/9806471.

[57] Konstantin Chetyrkin, Mikolaj Misiak, Manfred Munz, Phys.Lett. B400 (1997) 206219; Erratum-ibid. B425 (1998) 414 arXiv:hep-ph/9612313. 


\section{PROGRAM SUMMARY}

Manuscript Title: SUSY_FLAVOR: a computational tool for FCNC and CP-violating processes in the MSSM

Authors: J. Rosiek, P. H. Chankowski, A. Dedes, S. Jäger, P. Tanedo

Program Title: SUSY_FLAVOR

Journal Reference:

Catalogue identifier:

Licensing provisions: None

Programming language: Fortran 77

Operating system: Any, tested on Linux

Keywords: Supersymmetry, $K$ physics, $B$ physics, rare decays, CP-violation

PACS: 12.60.Jv, 13.20.He

Classification: 11.6 Phenomenological and Empirical Models and Theories

Nature of problem:

Predicting CP-violating observables, meson mixing parameters and branching ratios for set of rare processes in the general R-parity conserving MSSM.

Solution method:

We use standard quantum theoretical methods to calculate Wilson coefficients in MSSM and at one loop including QCD corrections at higher orders when this is necessary and possible. The input parameters can be read from an external file in SLHA format.

Restrictions:

The results apply only to the case of MSSM with R-parity conservation.

Unusual features:

Running time:

For single parameter set approximately 1s in double precision on a PowerBook Mac G4

References:

[1] M. Misiak, S. Pokorski and J. Rosiek, "Supersymmetry and the FCNC effects" Adv. Ser. Direct. High Energy Phys. 15 (1998) 795 arXiv:hep-ph/9703442.

[2] S. Pokorski, J. Rosiek and C. A. Savoy, Nucl. Phys. B 570 (2000) 81 arXiv:hep-ph/9906206.

[3] A. J. Buras, P. H. Chankowski, J. Rosiek and L. Slawianowska, Nucl. Phys. B 619 (2001) 434 arXiv:hep-ph/0107048.

[4] A. J. Buras, P. H. Chankowski, J. Rosiek and L. Slawianowska, Nucl. Phys. B 659 (2003) 3 arXiv:hep-ph/0210145.

[5] A. J. Buras, T. Ewerth, S. Jager and J. Rosiek, Nucl. Phys. B 714 (2005) 103 arXiv:hep-ph/0408142.

[6] A. Dedes, J. Rosiek and P. Tanedo, Phys. Rev. D 79 (2009) 055006 arXiv:0812.4320 [hep$\mathrm{ph}]$. 